\title{
AN APPLICATION OF DOUBLY ORTHOGONAL FUNCTIONS TO A PROBLEM OF APPROXIMATION IN TWO REGIONS $\left({ }^{(}\right)$
}

\author{
BY \\ PHILIP DAVIS
}

1. Introduction. In the plane of the complex variable $z$, let there be given two regions $G$ and $B$ which are bounded and such that $\bar{G} \subset B$. The regions may be simply- or multiply-connected, but will be considered to satisfy certain additional conditions which will be formulated precisely in a subsequent paragraph. We shall designate by $L^{2}(G)$ the set of functions which are singlevalued and regular in $G$ and which possess a finite norm:

$$
\|f\|_{G}^{2}=\iint_{G}|f|^{2} d A<\infty .
$$

By $L^{2}(B)$, we shall designate the similarly defined class of functions over $B$. In the present paper, the following process of approximation is considered. Let a function $f \in L^{2}(G)$ and a number $M$ be given. We are interested in approximating $f$ on $\bar{G}$ by a function $f^{*} \in L^{2}(B)$ whose $B$-norm does not exceed $M$ :

$$
\left\|f^{*}\right\|_{B} \leqq M
$$

and such that

$$
\left\|f-f^{*}\right\|_{G .}=\text { minimum. }
$$

A closely related problem was first proposed by Walsh, and has been considered in a series of papers by Walsh $[11 ; 12]\left({ }^{2}\right)$, and by Walsh and Nilson $[8 ; 13]$. In these papers, the problem is discussed using an $L^{p}$ norm defined by a line integral over the contours which bound the region. The Tchebycheff norm, corresponding to $p=\infty$, has also been considered. The principal aim of these writers was to establish asymptotic expressions for the degree of convergence of the best approximating functions as $M \rightarrow \infty$, and it was shown that this degree is governed by the relation between the region of regularity of $f$ and the level lines of a harmonic measure of the boundary

Received by the editors May 10, 1951.

(1) Research Paper done under Navy Contract N5 ori-07634 at Harvard University under the direction of Professor J. L. Walsh. The ideas expressed in this paper represent the personal views of the author, and are not necessarily those of the Office of Naval Research.

The author is indebted to Professor Walsh for suggesting as a promising topic of research the relation between doubly orthogonal functions and the problem of approximation considered here, and wishes to express his thanks for numerous valuable suggestions in carrying out the present paper.

(2) Numbers in brackets refer to the bibliography at the end of the paper. 
of $B$ with respect to the difference region $B-G$.

In the present paper, we shall investigate an aspect of the problem which is more structural in nature. That is to say, we shall be interested in generating functions of best approximation, in discussing their totality, and in relating them to the domain functions which arise in the boundary value problem. The system of doubly orthogonal functions for the domains $G$ and $B$ introduced by Bergman $[1,2,3]$ plays a fundamental role in our development. These are a set of functions $\left\{\phi_{n}(z)\right\}(n=0,1, \cdots)$ which are of class $L^{2}(B)$, and are simultaneously orthogonal over $G$ and $B$ :

$$
\begin{gathered}
\iint_{G} \phi_{n}(z)\left(\phi_{m}(z)\right)^{-d A}=\delta_{m n} ; \\
\iint_{B} \phi_{n}(z)\left(\phi_{m}(z)\right)-d A=k_{m} \delta_{m n}, k_{m}>1,
\end{gathered}
$$

where $\left(\phi_{m}(z)\right)$-denotes the complex conjugate of $\phi_{m}(z)$. The connection is made using the Fredholm theory of integral equations. The latter theory will enable us to obtain an explicit representation of functions of best approximation by showing that they may be generated by a resolvent kernel which is closely related to both the kernel functions of the region $G$ and of the region $B$. It will appear, moreover, that the convergence of an $f\left(\in L^{2}(B)\right)$ of its functions of best approximation as $M \rightarrow \infty$ may be given an interpretation as a process of generalized Borel summation.

It should be recalled that the kernel function of a region $D$ has been defined (Bergman [3]) as

$$
K_{D}(z, \bar{w})=\sum_{n=0}^{\infty} p_{n}(z)\left(p_{n}(w)\right)^{-}
$$

where $\left\{p_{n}(z)\right\}$ is any closed orthonormal set over $D$ :

$$
\iint_{D} p_{m}(z)\left(p_{n}(z)\right)-d A=\delta_{m n}
$$

If $D$ is simply-connected and has a boundary $d$ which is such that all the points which do not lie in $D+d$ form a single region whose boundary is exactly $d$, then (Farrell [5]) a closed orthonormal set may be found by orthonormalizing the powers $1, z, z^{2}, \cdots$. For many multiply-connected regions, such a set may be found by orthonormalizing appropriately selected powers $\left(z-a_{k}\right)^{n}(n=0, \pm 1, \pm 2, \cdots ; k=1, \cdots, m)$. For the purposes of the present paper, we shall therefore consider the kernel functions of the regions dealt with as "known" functions. Actually, in what follows, we require only a knowledge of the kernel function of the larger domain, $K_{B}(z, \bar{w})$. If, in the case of simply-connected regions, a preliminary conformal map of $B$ 
onto the unit circle is made, then we have very simply $K_{B}(z, \bar{w})$ $=(1 / \pi)(1-z \bar{w})^{-2}$.

Our principal theorem, proved in $\S 4$, can be stated as follows.

TheOREM. Let $k^{*}(z, \bar{w} ; \lambda)$ designate the resolvent kernel of the integral equation

$$
f(z)=g(z)+\frac{1}{\lambda} \iint_{G} K_{B}(z, \bar{w}) g(w) d A_{w} .
$$

If $f(z) \in L^{2}(G)$, then

$$
f(z ; \lambda)=\iint_{G} k^{*}(z, \bar{w} ; \lambda) f(w) d A_{w}
$$

$i$ s, for each positive $\lambda$, a function of class $L^{2}(B)$ of best approximation in $G$ to $f(z)$.

Despite the fact that the doubly orthogonal functions $\phi_{n}(z)$ play a fundamental role in the theory, it is to be emphasized that the final results are independent of them. This is of particular importance in view of the relative inaccessibility of the doubly orthogonal functions corresponding to two arbitrary regions $G$ and $B$.

2. An extremal problem in Hilbert space. In the present section, we deal with the Hilbert space $l^{2}$ of sequences $A=\left\{a_{n}\right\}$ of complex numbers for which

$$
\|A\|^{2}=\sum_{n=0}^{\infty}\left|a_{n}\right|^{2}<\infty .
$$

Let there be given a fixed sequence of positive constants $k_{n}$ for which

$$
\sum_{n=0}^{\infty} \frac{1}{k_{n}}<\infty .
$$

By the hyperellipse $\varepsilon=\mathcal{E}\left(k_{n} ; M\right)$ we shall mean the set of points $A$ whose coordinates satisfy the inequality

$$
\sum_{n=0}^{\infty}\left|a_{n}\right|^{2} k_{n} \leqq M^{2} .
$$

By the surface of the hyperellipse $\mathcal{E}\left(k_{n} ; M\right)$ we shall mean the set of points $A$ for which equality in (2.3) holds. In the space $l^{2}$, we consider the problem of minimizing the distance from a given point $A^{*}$ to a given hyperellipse. It will appear subsequently that the characteristic properties of minimal distances in Euclidean $n$-spaces will carry over to this more general situation.

The set $\varepsilon$ is both convex and compact. To show the former, let $A=\left\{a_{n}\right\}$ and $B=\left\{b_{n}\right\}$ both belong to $\varepsilon$. Consider now the point $P=\sigma A+(1-\sigma) B$, $0<\sigma<1$. By Minkowski's inequality we have 


$$
\begin{aligned}
{\left[\sum_{n=0}^{\infty}\left|\sigma a_{n}+(1-\sigma) b_{n}\right|{ }^{2} k_{n}\right]^{1 / 2} } & \\
& \leqq \sigma\left[\sum_{n=0}^{\infty}\left|a_{n}\right|^{2} k_{n}\right]^{1 / 2}+(1-\sigma)\left[\sum_{n=0}^{\infty}\left|b_{n}\right|^{2} k_{n}\right]^{1 / 2} \\
& \leqq \sigma M+(1-\sigma) M=M .
\end{aligned}
$$

Therefore $P \in \mathcal{E}$. To show that $\varepsilon$ is compact, we may make use of the following well known necessary and sufficient condition for compactness in an $l^{p}$ space: a subset $S$ of $l^{p}$ is compact if and only if (a) there exist constants $m_{0}, m_{1}, \cdots$ such that for all $A \in S$ we have

$$
\left|a_{n}(A)\right| \leqq m_{n} \quad(n=0,1, \cdots)
$$

and (b) the series

$$
\sum_{n=0}^{\infty}\left|a_{n}(A)\right|^{p}
$$

converges uniformly for $A \in S$. Reverting now to $p=2$ and $S=\varepsilon$ we see firstly from (2.3) that for all $A \in \mathcal{E}\left(k_{n} ; M\right)$ we have $\sum_{n=0}^{\infty}\left|a_{n}(A)\right|^{2} k_{n} \leqq M^{2}$, so that a fortiori $\left|a_{n}(A)\right|{ }^{2} k_{n} \leqq M^{2}$ and hence

$$
\left|a_{n}(A)\right| \leqq M / k_{n}^{1 / 2} \quad(n=0,1, \cdots) .
$$

Thus condition (a) is satisfied. Secondly, by (2.6), the series $\sum_{n=0}^{\infty}\left|a_{n}(A)\right|^{2}$ is uniformly dominated by $\sum_{n=0}^{\infty} M^{2} / k_{n}<\infty$, in virtue of (2.1). Thus (b) is satisfied, and $\varepsilon$ is therefore compact.

Because of the compactness of $\mathcal{E}$, it follows from general topological considerations that, given any $A^{*} \in l^{2}$, there will exist a point $A \in \mathcal{E}$, such that $\left\|A^{*}-A\right\|$ is a minimum, that is, $\left\|A^{*}-A\right\|=$ g.l.b. $A^{\prime} \in \varepsilon\left\|A^{*}-A^{\prime}\right\|$. Furthermore, it is a consequence of the convexity of $\mathcal{E}$ that the point $A$, and hence also the minimizing vector $A^{*}-A$, are uniquely determined. For, suppose that there are two distinct points $A$ and $A^{\prime}$ such that $\left\|A^{*}-A\right\|=\left\|A^{*}-A^{\prime}\right\|$ $=m=$ minimum. The point $B=\left(A+A^{\prime}\right) / 2$ is in $\mathcal{E}$. Also, $\left\|A^{*}-B\right\|^{2}$ $=(1 / 4)\left\|\left(A^{*}-A\right)+\left(A^{*}-A^{\prime}\right)\right\|^{2}$. Now for arbitrary $X, Y \in l^{2}$ we have $\|X+Y\|^{2}$ $+\|X-Y\|^{2}=2\|X\|^{2}+2\|Y\|^{2}, \quad$ so that $\left\|A^{*}-B\right\|^{2}=(1 / 2)\left\|A^{*}-A\right\|^{2}+(1 / 2)$ . $\left\|A^{*}-A^{\prime}\right\|^{2}-(1 / 4)\left\|\left(A^{*}-A\right)-\left(A^{*}-A^{\prime}\right)\right\|^{2}=m^{2}-(1 / 4)\left\|A-A^{\prime}\right\|^{2}<m^{2}$. Thus, the assumption that both $A$ and $A^{\prime}$ are minimal is contradicted.

We may therefore write $A\left(A^{*} ; M\right)$ and $\Delta\left(A^{*} ; M\right)$ to designate unambiguously the minimal point and minimal distance from $A^{*}$ to $\mathcal{E}\left(k_{n} ; M\right)$. For a given point $A^{*}=\left\{a_{n}^{*}\right\}$, the series $\sum_{n=0}^{\infty}\left|a_{n}^{*}\right|^{2} k_{n}$ may either converge or diverge to plus infinity. In the former case, $A^{*} \in \mathcal{E}\left(k_{n} ; M\right)$ for $M$ sufficiently large, and we shall have $\Delta\left(A^{*}, M\right)=0$ for $M$ sufficiently large. However, even in the latter case (of divergence), we have, as we shall immediately prove, 


$$
\lim _{M \rightarrow \infty} \Delta\left(A^{*}, M\right)=0 .
$$

It thus appears that any family of hyperellipses $\varepsilon\left(k_{n} ; M_{j}\right)$ for which lim $\sup _{j \rightarrow \infty} M_{j}=\infty$ must be dense in the space $l^{2}$. To prove (2.7), we must show that to a given $\epsilon>0$, there exists an $M_{\epsilon}$ such that $\left\|A^{*}-A\left(A^{*}, M\right)\right\| \leqq \epsilon$ for all $M \geqq M_{\epsilon}$. Since $A^{*} \in l^{2}$, we may determine an $n=n_{\epsilon}$ such that $\sum_{n+1}^{\infty}\left|a_{i}{ }^{*}\right|^{2} \leqq \epsilon^{2}$. With this $n$, let $M_{\epsilon}=\left[\sum_{j=0}^{n}\left|a_{j}^{*}\right|{ }^{2} k_{j}\right]^{1 / 2}$. If, now, $P=P(\epsilon)$ is the point with coordinates $\left\{a_{0}^{*}, a_{1}^{*}, \cdots, a_{n_{\varepsilon}}^{*}, 0,0,0, \cdots\right\}$, then we have $P \in \mathcal{E}\left(k_{n} ; M\right)$ for $M \geqq M_{\epsilon}$. Finally, $\left\|A^{*}-A\left(A^{*} ; M\right)\right\| \leqq\left\|A^{*}-P\right\|=\epsilon$.

It is a consequence of the denseness of the family $\mathcal{E}\left(k_{n} ; M_{j}\right)$ that if $A^{*} \notin \mathcal{E}\left(k_{n} ; M\right)$, then the minimizing point $A\left(A^{*}, M\right)$ must lie on the surface of $\varepsilon$. For, suppose that $A=\left\{a_{n}\right\}$ and $\sum_{n=0}^{\infty}\left|a_{n}\right|^{2} k_{n}=M_{1}<M$. If $A^{*}$ $\notin \mathcal{E}\left(k_{n} ; M\right)$, then by the previous result (2.7) we may find an $M_{2}$ sufficiently large so that $\Delta\left(A^{*}, M_{2}\right)<\Delta\left(A^{*}, M\right)$. Consider now the point $A^{\prime}=\left\{a_{n}^{\prime}\right\}$ $=(1-\sigma) A\left(A^{*}, M\right)+\sigma A\left(A^{*}, M_{2}\right)$, where $0<\sigma<\left(M-M_{1}\right) /\left(M_{2}-M_{1}\right)<1$. Since $\sum_{n=0}^{\infty}\left|a_{n}{ }^{\prime}\right|{ }^{2} k_{n} \leqq(1-\sigma) M_{1}+\sigma M_{2}<M$, we have $A^{\prime} \in \mathcal{E}\left(k_{n} ; M\right)$. But also, $\left\|A^{*}-A^{\prime}\right\|=(1-\sigma) \Delta\left(A^{*}, M\right)+\sigma \Delta\left(A^{*}, M_{2}\right)<\Delta\left(A^{*}, M\right)$, so that we have obtained a contradiction, for by assumption, $A$ is the minimum point from $A^{*}$ to $\mathcal{E}\left(k_{n} ; M\right)$.

We turn now to the question of obtaining explicit representations for the quantities $A\left(A^{*} ; M\right)$ and $\Delta\left(A^{*} ; M\right)$. Such representations are possible to obtain by means of a limiting process from the case of finite-dimensional spaces. By way of a preliminary result, we establish the following lemma which gives the solution for the finite-dimensional case.

Lemma. Let $M>0$, and $n$, a fixed positive integer, be given. Let also the point $A^{*}=\left\{a_{0}^{*}, a_{1}{ }^{*}, \cdots, a_{n}^{*}\right\}$ be assumed to lie exterior to $\mathcal{E}\left(k_{0}, k_{1}, \cdots, k_{n} ; M\right)$. Then the extremal problem

$$
\sum_{j=0}^{n}\left|a_{i}^{*}-a_{j}\right|^{2}=\text { minimum }
$$

under the condition

$$
\sum_{j=0}^{n}\left|a_{j}\right|^{2} k_{j} \leqq M^{2}
$$

is solved by the quantities

$$
a_{j}=a_{i}^{*} /\left(1+\lambda k_{j}\right) \quad(j=0,1, \cdots, n)
$$

where $\lambda$ is the positive number determined by

$$
\sum_{j=0}^{n}\left|a_{j}^{*}\right|^{2} k_{j} /\left(1+\lambda k_{j}\right)^{2}=M^{2} .
$$


Proof. Let us first note that by our previous reasoning, the minimal point will lie on the surface of $\varepsilon$, that is, equality must hold in (2.9). We note further that if the left side of $(2.11)$ be designated by $Q(\lambda)$, then $Q(\lambda)$ is a continuous, monotonically decreasing function of $\lambda$, tending to 0 as $\lambda \rightarrow \infty$. Since $A^{*}$ is exterior to $\mathcal{E}, \sum_{i=0}^{n}\left|a_{i}^{*}\right|{ }^{2} k_{i}=Q(0)>M^{2}$, so that (2.11) has a unique positive solution. If we now write $a_{j}=\alpha_{j}+i \alpha_{n+j+1} ; a_{j}^{*}=\alpha_{j}^{*}+i \alpha_{n+j+1}^{*}$, $k_{j}^{\prime}=k_{j}(j=0,1, \cdots, n) ; k_{n+j+1}^{\prime}=k_{j}(j=0,1, \cdots, n)$, then our problem is reduced to the (real) problem of minimizing

$$
\sum_{j=0}^{2 n+1}\left(\alpha_{j}^{*}-\alpha_{j}\right)^{2}
$$

under the conditions

$$
\sum_{j=0}^{2 n+1}\left(\alpha_{j}\right)^{2} k_{j}^{\prime}=M^{2}
$$

Introducing a Lagrange multiplier $\lambda$, we have $2\left(\alpha_{j}-\alpha_{j}^{*}\right)=2 \lambda k_{j}^{\prime} \alpha_{j}=0$ $(j=0,1, \cdots, 2 n+1)$ so that

$$
\alpha_{j}=\alpha_{i}^{*} /\left(1+\lambda k_{j}\right) \quad(j=0,1, \cdots, 2 n+1)
$$

and hence

$$
a_{i}=a_{i}^{*} /\left(1+\lambda k_{j}\right) \quad(j=0,1, \cdots, n) .
$$

From the very structure of the problem, it follows that sgn $a_{j}=\operatorname{sgn} a_{j}^{*}$ $(j=0,1, \cdots, n)$. Therefore $1+\lambda k_{j}>0$, so that $\lambda>\max \left(-1 / k_{j}\right)$. Since $Q^{\prime}(\lambda)$ $=-\sum_{i=0}^{n} 2\left|a_{i}^{*}\right|{ }^{2} k_{i}^{2} /\left(1+\lambda k_{i}\right)^{3}$, it follows that $Q^{\prime}(\lambda)<0$ for all $\lambda>\max \left(-1 / k_{j}\right)$. It is therefore clear that $\lambda$ must be positive. Finally, (2.11) follows from (2.9) and (2.10).

Theorem 1. Let $A^{*}=\left\{a_{j}^{*}\right\} \in l^{2}, A^{*} \notin \mathcal{E}\left(k_{n} ; M\right)$. If $\left\{a_{j}\right\}=A\left(A^{*}, M\right)$, then we must have

$$
a_{i}=a_{i}^{*} /\left(1+\lambda k_{i}\right) \quad(j=0,1, \cdots)
$$

where $\lambda$ is the unique positive root of

$$
Q(\lambda)=\sum_{j=0}^{\infty}\left|a_{j}^{*}\right|{ }^{2} k_{j} /\left(1+\lambda k_{j}\right)^{2}=M^{2} .
$$

Proof. The function $Q(\lambda)$ is clearly analytic in the right half of the complex $\lambda$-plane, decreasing to 0 monotonically as $\lambda \rightarrow+\infty$. Since $A^{*} \in \mathcal{E}\left(k_{n} ; M\right)$, we have $Q(0)>M^{2}$ so that (2.16) possesses a unique positive solution. We may observe further that as $M \rightarrow \infty, \lambda=\lambda(M) \rightarrow 0$.

For each positive integer $n$, let $A_{n}$ designate the point of $l^{2}$ whose coordinates are given by 


$$
A_{n}=\left\{a_{0}^{*} /\left(1+\lambda_{n} k_{0}\right), \cdots, a_{n}^{*} /\left(1+\lambda_{n} k_{n}\right), 0,0,0, \cdots\right\}
$$

where the quantity $\lambda_{n}$ has been determined as in the preceding lemma. It may easily be verified that as $n \rightarrow \infty, \lambda_{n}$ increases monotonically to $\lambda$. Let $A^{\prime}$ designate the point $\left\{a_{j}^{*} /\left(1+\lambda k_{j}\right)\right\}$. We have $\lim _{n \rightarrow \infty}\left\|A^{\prime}-A_{n}\right\|=0$. For,

$$
\begin{aligned}
\left\|A_{n}-A^{\prime}\right\|^{2}= & \left(\lambda-\lambda_{n}\right)^{2} \sum_{j=0}^{n}\left|k_{j}\right|^{2} a_{j}^{* 2} /\left(1+\lambda k_{j}\right)^{2}\left(1+\lambda_{n} k_{j}\right)^{2} \\
& +\sum_{j=n+1}^{\infty}\left|a_{j}^{*}\right|^{2} /\left(1+\lambda k_{j}\right) \\
\leqq & \left(\lambda-\lambda_{n}\right)^{2} / \lambda_{n}^{4} \sum_{j=0}^{\infty}\left|a_{j}^{*}\right|^{2} / k_{j}^{2}+o(1) \quad(n \rightarrow \infty) .
\end{aligned}
$$

By our previous discussion, we know that the minimum problem has a unique solution $A=\left\{a_{j}\right\}$. Let $B_{n}$ designate the point of $l^{2}$ whose coordinates are given by

$$
B_{n}=\left\{a_{0}, a_{1}, \cdots, a_{n}, 0,0,0, \cdots\right\} .
$$

Now, $\left\|A^{*}-B_{n}\right\|^{2}=\sum_{j=0}^{n}\left|a_{j}^{*}-a_{j}\right|^{2}+\sum_{j=n+1}^{\infty}\left|a_{j}^{*}\right|^{2}$, hence $\lim _{n \rightarrow \infty}\left\|A^{*}-B_{n}\right\|^{2}$ $=\Delta^{2}\left(A^{*}, M\right)$. Now, $\Delta \leqq\left\|A^{*}-A_{n}\right\|$ and, by our lemma, $\left\|A^{*}-A_{n}\right\|$ $\leqq\left\|A^{*}-B_{n}\right\|$, whence

$$
\Delta \leqq\left\|A^{*}-A^{\prime}\right\|+\left\{\left\|A^{*}-A_{n}\right\|-\left\|A^{*}-A^{\prime}\right\|\right\} \leqq\left\|A^{*}-B_{n}\right\| .
$$

Since the bracket is in absolute value less than $\left\|A^{\prime}-A_{n}\right\|$, it tends to 0 , leaving $\left\|A^{*}-A^{\prime}\right\|=\Delta$. By the uniqueness of the minimal point, we must have $A^{\prime}=A$, and therefore (2.15) holds.

\section{Corollary.}

$$
\Delta^{2}\left(A^{*} ; M\right)=\lambda^{2} \sum_{j=0}^{\infty}\left|a_{j}^{*}\right|^{2} k_{j}^{2} /\left(1+\lambda k_{j}\right)^{2} .
$$

It is clear moreover that $\Delta^{2}$ considered as a function of the complex variable $\lambda$ is analytic for $\operatorname{Re} \lambda \geqq 0$. It is of considerable interest to obtain a geometric interpretation of the solution (2.15). In the finite-dimensional case, the minimal vector is normal to the surface of the hyperellipse. The equations (2.15) imply that this is also true for the space $l^{2}$. Let the point $A$ $=\left\{a_{i}\right\}$ lie on the surface of $\mathcal{E}\left(k_{i} ; M\right)$, and let $Y=\left\{y_{i}\right\}, Y \neq 0$, be a point of $l^{2}$, and such that

$$
\sum_{i=0}^{\infty} k_{i} a_{i} \bar{y}_{i}=0
$$

The line 


$$
P(\sigma)=A+\sigma Y=\left\{p_{i}\right\},
$$

$\sigma$ complex,

will be a tangent to $\mathcal{\varepsilon}\left(k_{i} ; M\right)$. For $P(0)$ lies on $\varepsilon$, while if $\sigma \neq 0$ we have by (2.19)

$$
\sum_{i=0}^{\infty}\left|p_{i}\right|^{2} k_{i}=\sum_{i=0}^{\infty}\left|a_{i}\right|^{2} k_{i}+|\sigma|^{2} \sum_{i=0}^{\infty}\left|y_{i}\right|{ }^{2} k_{i}>M^{2}
$$

Thus, $P(\sigma) \notin \varepsilon$.

CoRollary. The minimal vector $A^{*}-A$ is orthogonal to any tangent vector issuing from $A$, that is, to any vector of the form $\sigma Y$, where $Y$ is subject to (2.19). More explicitly, (2.19) implies

$$
\sum_{i=0}^{\infty}\left(a_{i}^{*}-a_{i}\right) \bar{y}_{i}=0 .
$$

Proof. $A^{*}-A=\left\{a_{i}^{*} /\left(1+\lambda k_{i}\right)-a_{i}^{*}\right\}, \quad \sigma Y=\left\{\sigma y_{i}\right\} \quad$ where $y_{i}$ satisfy $\sum_{i=0}^{\infty} a_{i}^{*} k_{i} \bar{y}_{i} /\left(1+\lambda k_{i}\right)=0$. Thus, $\left(A^{*}-A, \quad \sigma Y\right)=\bar{\sigma} \sum_{i=0}^{\infty} \quad\left[a_{i}^{*} \bar{y}_{i} /\left(1+\lambda k_{i}\right)\right.$ $\left.-a_{i} \bar{y}_{i}\right]=-\bar{\sigma} \lambda \sum_{i=0}^{\infty} a_{i}^{*} k_{i} \bar{y}_{i} /\left(1+\lambda k_{1}\right)=0$.

A geometric interpretation of the multiplier $\lambda$ may be obtained as follows. From (2.16) there is obtained

$$
-\lambda d M^{2} / d \lambda=2 \lambda \sum_{j=0}^{\infty}\left|a_{j}^{*}\right|^{2} k_{j}^{2} /\left(1+\lambda k_{j}\right)^{3}
$$

while from (2.18),

$$
\begin{aligned}
d \Delta^{2} / d \lambda & =-2 \lambda^{2} \sum_{j=0}^{\infty}\left|a_{j}^{*}\right|^{2} k_{j}^{3} /\left(1+\lambda k_{j}\right)^{3}+2 \lambda \sum_{j=0}^{\infty}\left|a_{j}^{*}\right|^{2} k_{j}^{2} /\left(1+\lambda k_{j}\right)^{2} \\
& =2 \lambda \sum_{j=0}^{\infty}\left|a_{j}^{*}\right|^{2} k_{j}^{2} /\left(1+\lambda k_{j}\right)^{3} .
\end{aligned}
$$

Thus we find

$$
\lambda=-(\Delta / M) d \Delta / d M .
$$

3. The transformation $T$. Let the region $B$ lying in the complex $z$-plane possess the kernel function $K_{B}(z, w \bar{v})$. In addition, let there be given a region $G$ for which $\bar{G} \subset B$. In preparation for what follows, we shall introduce a transformation $T$ to be applied to functions $f \in L^{2}(G)$ and given by

$$
T f(z)=\iint_{G} K_{B}(z, \bar{w}) f(w) d A_{w} .
$$

In view of the condition $\bar{G} \subset B$, the kernel $K_{B}(z, w)$ is regular over $G$. The operator $T$ acts as a stretching in the sense that the transforms $T f$ belong 
to $L^{2}(B)$. For, let $\left\{p_{n}(z)\right\}$ be a set of functions which are closed and orthonormal over $B$. Then by (1.5) we may write

$$
T f(z)=\iint_{G} \sum_{n=0}^{\infty} p_{n}(z)\left(p_{n}(w)\right)^{-f(w)} d A_{w}
$$

so that

$$
T f(z)=\sum_{n=0}^{\infty} \alpha_{n} p_{n}(z) \text { where } \quad \alpha_{n}=\iint_{G} f(w)\left(p_{n}(w)\right)-d A_{w} .
$$

By the Schwarz inequality we have

$$
\left|\alpha_{n}\right|^{2} \leqq\|f\|_{G}^{2}\left\|p_{n}\right\|_{G}^{2}
$$

and therefore

$$
\sum_{n=0}^{\infty}\left|\alpha_{n}\right|^{2} \leqq\|f\|_{a}^{2} \iint_{G} K_{B}(w, \bar{w}) d A_{w}<\infty .
$$

It follows by the Riesz-Fischer Theorem for the class $L^{2}(B)$ (cf. Bergman $\left[3\right.$, p. 5]) that $T f(z) \in L^{2}(B)$.

In particular, let $w$ be fixed in $G$. Then $T$ acting on $K_{G}(z, \bar{w})$ yields the kernel function of $B$ :

$$
T K_{G}(z, \bar{w})=K_{B}(z, \bar{w}) .
$$

For, $T K_{G}(z, \bar{w})=\iint_{G} K_{B}(z, \bar{t}) K_{G}(t, \bar{w}) d A_{t}$, and hence (3.4) follows by the reproducing property of the kernel $K_{G}$ over $G$. If the boundary of $B$ is sufficiently smooth, then the operator $T$ will stretch $f$ beyond the confines of the region $B$. More precisely, we have the following result.

THEOREM 2. Let the region $B$ be bounded by closed analytic curves. Then there will exist a region $B^{*}$ such that $\bar{B} \subset B^{*}$ and such that all transforms $T f$ of functions $f \in L^{2}(G)$ are regular in $B^{*}$.

Proof. Introduce $g_{B}(z, w)=g_{B}(x, y ; u, v), z=x+i y, w=u+i v$, the Green's function of the region $B$. It is known (cf. Schiffer [9], Bergman [3, p. 58]) that

$$
K_{B}(z, \bar{w})=-\frac{2}{\pi} \frac{\partial^{2} g_{B}(z, w)}{\partial z \partial \bar{w}}
$$

where

$$
\frac{\partial}{\partial z}=\frac{1}{2}\left(\frac{\partial}{\partial x}-i \frac{\partial}{\partial y}\right), \quad \frac{\partial}{\partial \bar{z}}=\frac{1}{2}\left(\frac{\partial}{\partial x}+i \frac{\partial}{\partial y}\right)
$$

and we may therefore write, as an alternate form of (3.1), 


$$
T f(z)=-\frac{2}{\pi} \iint_{G} \frac{\partial^{2} g_{B}(z, w)}{\partial z \partial \bar{w}} f(w) d A_{w} .
$$

Let $w$ be a fixed point of $G$. Inasmuch as the boundary of $B$ is analytic, $g_{B}(z, w)$ is a harmonic function of $x, y$ in $\bar{B}$, and hence $\partial^{2} g_{B}(z, w) / \partial z \partial \bar{w}$ is analytic for $z \in \bar{B}$. To each boundary point $z_{b}$ of $B$ there is a circle $\left|z-z_{b}\right|$ $<\rho, \rho=\rho\left(z_{b}, w\right)$, in which $K_{B}(z, \bar{w})$ is analytic, and hence, by the compactness of $\bar{G}$, for each boundary point $z_{b}$ there is a positive $\rho=\rho\left(z_{b}\right)$ such that $K_{B}(z, \bar{w})$ is regular in $\left|z-z_{b}\right|<\rho$ for all $w \in \bar{G}$. We may cover the boundary with a finite number of such circles. If these are designated by $C_{i}$ $(i=1,2, \cdots, n)$, and if $B^{*}=B+\sum_{i=1}^{n} C_{i}$, we shall have $K_{B}(z, \bar{w})$ regular in $B^{*}$ for all $w \in \bar{G}$. Therefore, by (3.1), $T f(z)$ must be regular for $z \in B^{*}$.

It should be noted that the character of the boundary of $G$ plays no role in this result qualitatively, but of course serves to determine the best possible $B^{*}$. If, for instance, $B$ is the unit circle, we have

$$
K_{B}(z, \bar{w})=(1 / \pi)(1-z \bar{w})^{-2}
$$

so that the transformation $T$ takes the particularly simple form

$$
T f(z)=\frac{1}{\pi} \iint_{G} \frac{f(w) d A_{w}}{(1-z \bar{w})^{2}} .
$$

From this follows that if $G$ is simply-connected and contains the origin, then the transforms $T f$ will all be regular in $G^{I}$, the region containing the origin and bounded by the reflection in $B$ of the boundary of $G$.

The iterates of $T$ are defined by

$$
\begin{aligned}
T^{(n)} f(z) & =\iint_{G} K_{B}(z, \bar{w}) T^{(n-1)} f(w) d A_{w} \quad(n=1,2, \cdots), \\
T^{0} & =1 .
\end{aligned}
$$

They may be written in the alternate form

$$
T^{(n)} f(z)=\iint_{G} K_{B}^{(n)}(z, \bar{w}) f(w) d A_{w}
$$

where the iterated kernels have been defined by

$$
\begin{aligned}
K_{B}^{(1)}(z, \bar{w}) & =K_{B}(z, \bar{w}) ; \\
K_{B}^{(n)}(z, \bar{w}) & =\iint_{G} K_{B}(z, \bar{t}) K_{B}^{(n-1)}(t, \bar{w}) d A_{t} \quad(n=2,3, \cdots) .
\end{aligned}
$$

It should be observed that for $f \in L^{2}(G)$, the iterated transforms $T^{(n)} f(z)$ $(n \geqq 1)$ are all of class $L^{2}(B)$, and if $B$ is bounded by analytic curves, will be regular in a larger region $B^{*}$. 
4. An integral equation of Fredholm type. Let the regions $G$ and $B$ be given such that $\bar{G} \subset B$, and let their respective kernel functions be $K_{G}(z, \bar{w})$ and $K_{B}(z, \bar{w})$. We construct the following linear combination of the kernels $K_{G}$ and $K_{B}$ :

$$
K(z, \bar{w} ; \lambda)=K_{G}(z, \bar{w})-\lambda K_{B}(z, \bar{w})
$$

where $\lambda$ is a complex variable. It is clear that $K$ is regular for all $z, w \in G$ and for all $\lambda$. We next form the integral equation of Fredholm type

$$
g(z)=\iint_{G} K(z, \bar{w} ; \lambda) f(w) d A_{w}, \quad f \in L^{2}(G) .
$$

This may be written in the more familiar form

$$
\begin{array}{rlr}
g(z) & =f(z)-\lambda \iint_{G} K_{B}(z, \bar{w}) f(w) d A_{w} & \\
& =(I-\lambda T) f(z) & (I f=f) .
\end{array}
$$

We shall be interested in (4.3) both from the Fredholm and Hilbert-Schmidt points of view. A formal solution of (4.3) is given by the Neumann series

$$
f(z)=(I-\lambda T)^{-1} g(z)=\sum_{n=0}^{\infty} \lambda^{n} T^{(n)} g(z)
$$

and this solution will be valid for values of $\lambda$ which are sufficiently small in absolute value. We may rewrite (4.4) in the form

$$
f(z)=g(z)+\lambda \iint_{G} k(z, \bar{w} ; \lambda) g(w) d A_{w}
$$

where

$$
k(z, \bar{w} ; \lambda)=\sum_{n=1}^{\infty} \lambda^{n-1} K_{B}^{(n)}(z, \bar{w}) .
$$

The Fredholm resolvent is (for each fixed $z, w \in G$ ) known to be a meromorphic function of $\lambda$, and may be expressed as the ratio of two entire functions of $\lambda$ in the following manner.

$$
k(z, \bar{w} ; \lambda)=D(z, \bar{w} ; \lambda) / D(\lambda)
$$

where $D(z, \bar{w} ; \lambda)$ and $D(\lambda)$ have the following power series representations, each converging for $|\lambda|<\infty$ :

$$
D(z, \bar{w} ; \lambda)=\sum_{n=0}^{\infty} \frac{(-1)^{n} \lambda^{n} A_{n}(z, \bar{w})}{n !},
$$




$$
D(\lambda)=\sum_{n=0}^{\infty} \frac{(-1)^{n} c_{n} \lambda^{n}}{n !} .
$$

The functions $A_{n}(z, \bar{v})$ and the constants $c_{n}$ may be expressed by recursion as follows

$$
\begin{aligned}
A_{0}(z, \bar{w}) & =K_{B}(z, \bar{w}), \\
A_{n}(z, \bar{w}) & =c_{n} K_{B}(z, \bar{w})-n \iint_{G} A_{n-1}(z, \bar{t}) K_{B}(t, \bar{w}) d A_{t}, \\
c_{0} & =1 ; \quad c_{n+1}=\iint_{G} A_{n}(z, \bar{z}) d A_{z} .
\end{aligned}
$$

Closed expressions can be given for these quantities in terms of certain determinants. It is clear from (4.10a) and (4.10b) that each function $A_{n}$ is regular for $z, w \in B$. With $w$ fixed in $B$, and to each closed subdomain of the regularity domain of $K_{B}(z, \bar{w})$, we may surely find an $m$ such that $\left|K_{B}(z, \bar{w})\right|$ $\leqq m$ holds for all $z$ in that subdomain. The Fredholm theory then tells us that the functions $A_{n}$ will satisfy the inequality

$$
\left|A_{n}(z, \bar{w})\right| \leqq m^{n+1}(n+1)^{(n+1) / 2} g^{n}
$$

where $g=\iint_{G} d A$. Thus, for all $\lambda$, the series (4.8) will converge uniformly and absolutely in every closed subregion $\bar{B}^{\prime} \subset B$, and will therefore be regular for $z, w \in B$.

The functions $A_{n}$ as well as the constants $c_{n}$ depend merely upon the domains $G$ and $B$, and are obtained by repeated integration of the kernel function of the larger domain over the smaller domain. Each $A_{n}$ is a linear combination of the iterated kernels $K_{B}, K_{B}^{(2)}, \cdots, K_{B}^{(n)}$. The constants $c_{n}$ are further related by the recursion formula

$$
c_{n+1}=\sum_{k=0}^{n}(-1)^{n-k} n ! a_{n-k+1} c_{k} / k ! ; \quad c_{0}=1,
$$

where

$$
a_{m}=\iint_{G} K_{B}^{(m)}(z, \bar{z}) d A_{z} .
$$

Referring to (4.5), we may write the solution of (4.3) in the form

$$
\begin{aligned}
f(z) & =g(z)+\frac{\lambda}{D(\lambda)} \iint_{G} D(z, \bar{w} ; \lambda) g(w) d A_{w} \\
& =g(z)+\frac{\lambda}{D(\lambda)} \sum_{n=0}^{\infty} \frac{(-1)^{n} \lambda^{n}}{n !} \iint_{G} A_{n}(z, \bar{w}) g(w) d A_{w} .
\end{aligned}
$$

This solution is valid for all values of $\lambda$ providing only that $D(\lambda) \neq 0$. These 
latter values are, of course, the eigenvalues of the homogeneous integral equation, and it is to this that we now turn. We consider, therefore, the equation

$$
f(z)=\lambda \iint_{G} K_{B}(z, \bar{w}) f(w) d A_{w}=\lambda T f(z) .
$$

In this connection, the following theorem due to S. Bergman $[3, \mathrm{p} .14]$ is of fundamental importance.

THEOREM 3. Let the region $B$ be bounded and let $\bar{G} \subset B$. Then there exists a system of functions $\left\{\phi_{n}(z)\right\}(n=0,1, \cdots)$ which are of class $L^{2}(B)$, and are such that

$$
\begin{array}{lr}
\iint_{B} \phi_{m}(z)\left(\phi_{n}(z)\right)-d A=k_{n} \delta_{m n}, & k_{n}>1, \\
\iint_{G} \phi_{m}(z)\left(\phi_{n}(z)\right)-d A=\delta_{m n} & (m, n=0,1, \cdots) .
\end{array}
$$

The system $\left\{\phi_{n}(z)\right\}$ is closed with respect to the class $L^{2}(B)$, and the constants $k_{n}$ satisfy the inequality

$$
\sum_{n=0}^{\infty} \frac{1}{k_{n}}<\infty .
$$

The above system of functions has been termed by Bergman a doubly orthogonal set for the regions $G$ and $B$. We may obviously assume that $k_{0} \leqq k_{1} \leqq k_{2} \leqq \cdots$. In view of $(4.16)$, the functions

$$
\phi_{n}^{*}(z)=\phi_{n}(z) / k_{n}^{1 / 2} \quad(n=0,1, \cdots)
$$

form a closed orthonormal set of functions for the region $B$ so that by (1.5) we have the following expression for the kernel function of $B$ :

$$
K_{B}(z, \bar{w})=\sum_{n=0}^{\infty} \phi_{n}(z)\left(\phi_{n}(w)\right)^{-/ k_{n}}
$$

convergent uniformly and absolutely for $z, w \in \bar{B}^{\prime} \subset B$. We may therefore write the homogeneous equation (4.15) in the form

$$
f(z)=\lambda \sum_{n=0}^{\infty} \frac{\phi_{n}(z)}{k_{n}} \iint_{G}\left(\phi_{n}(w)\right)^{-} f(w) d A .
$$

If we now make the selection $f(z)=\phi_{j}(z) ; \lambda=k_{j}(j=0,1, \cdots)$, then in view of (4.17), equation (4.21) will be satisfied identically. It thus appears that the doubly orthogonal functions $\left\{\phi_{n}(z)\right\}$ and the constants $k_{n}$ are respectively eigenfunctions and the corresponding eigenvalues of (4.15). 
The doubly orthogonal system $\left\{\phi_{n}(z)\right\}$ need not be closed with respect to $L^{2}(G)$, and in the general case will fail to be so. However, there is a wide class of regions $G, B$ for which we shall have closure over $L^{2}(G)$. This will be the case (cf. Bergman [3, p. 17]) whenever $B$ and $G$ are each bounded by a finite number of distinct Jordan curves and the inner region $G$ is such that it separates no point of $B-G$ from the boundary of $B$. In some cases, the requirement of a Jordan boundary can be weakened to the type of boundary studied by Farrell [5].

In the work which follows, we shall assume that the doubly orthogonal set is complete with respect to $L^{2}(G)$. In this case, we can assert that the system $\left\{\phi_{n}(z)\right\}$ constitutes the set of eigenfunctions for (4.15). In addition, we shall have

$$
K_{G}(z, \bar{w})=\sum_{n=0}^{\infty} \phi_{n}(z)\left(\phi_{n}(w)\right)^{-}
$$

convergent uniformly and absolutely in every $\bar{G}^{\prime} \subset G$. Returning to the nonhomogeneous equation (4.2) we have

$$
K(z, \bar{w} ; \lambda)=\sum_{n=0}^{\infty}\left(1-\frac{\lambda}{k_{n}}\right) \phi_{n}(z)\left(\phi_{n}(w)\right)^{-} .
$$

If, now, an $f(z) \in L^{2}(G)$ is given, it has a Fourier development

$$
f(z)=\sum_{n=0}^{\infty} a_{n} \phi_{n}(z) ; \quad \sum_{n=0}^{\infty}\left|a_{n}\right|^{2}<\infty
$$

convergent uniformly and absolutely for every $\bar{G}^{\prime} \subset G$, so that (4.2) becomes

$$
g(z)=\sum_{n=0}^{\infty}\left(1-\frac{\lambda}{k_{n}}\right) a_{n} \phi_{n}(z)
$$

and we have therefore

$$
f(z)=g(z)+\lambda \iint_{G} k(z, \bar{w} ; \lambda) g(w) d A_{w}
$$

where

$$
k(z, \bar{w} ; \lambda)=\sum_{n=0}^{\infty} \frac{\phi_{n}(z)\left(\phi_{n}(w)\right)^{-}}{k_{n}-\lambda} .
$$

Now for $\lambda \neq k_{n}(n=0,1, \cdots)$, we have $\left|1 /\left(k_{n}-\lambda\right)\right| \leqq \mu / k_{n}(n=0,1, \cdots)$ for some positive constant $\mu$, so that in view of (4.18), (4.20) the series (4.27) converges uniformly and absolutely for each fixed $w \in B$, and $z$ in $\bar{B}^{\prime} \subset B$, and represents a function of $z$ which is of class $L^{2}(B)$. Similarly in $w$. In view of the uniqueness of the resolvent kernel, we may identify the ex- 
pression (4.27) and that given by the Fredholm development (4.7).

From (3.12), (4.17), and (4.20) there is obtained

$$
K_{B}^{(n)}(z, \bar{w})=\sum_{j=0}^{\infty} \frac{\phi_{j}(z)\left(\phi_{j}(w)\right)^{-}}{k_{j}^{n}} \quad(n=1,2, \cdots)
$$

while from (3.12), (4.17), and (4.20) we have

$$
T^{(n)} f(z)=\sum_{j=0}^{\infty} \frac{a_{j} \phi_{j}(z)}{k_{j}^{n}} \quad(n=1,2, \cdots) .
$$

From (4.13), (4.17), and (4.28) there is obtained

$$
a_{n}=\sum_{j=0}^{\infty} k_{j}^{-n} .
$$

In addition to the resolvent kernel $k(z, \bar{w} ; \lambda)$, we shall find it convenient to deal with the closely related kernel given by

$$
k^{*}(z, \bar{w} ; \lambda)=\lambda^{-1} k\left(z, \bar{w} ;-\lambda^{-1}\right)=\sum_{n=0}^{\infty} \frac{\phi_{n}(z)\left(\phi_{n}(w)\right)^{-}}{1+\lambda k_{n}} .
$$

For each $z, w \in B, k^{*}(z, \bar{w} ; \lambda)$ is an analytic function whose only singularities are poles at $\lambda=-\left(k_{n}\right)^{-1}$, and which therefore induce an additional essential singularity of the second kind at $\lambda=0$. Since we have $k_{n}>1$, the singularities of $k^{*}(z, \bar{w} ; \lambda)$ considered as a function of $\lambda$ are therefore confined to the segment $-1>\operatorname{Re} \lambda \leqq 0$.

For fixed $\lambda \neq 0, \neq-k_{n}^{-1}$ and $w \in B, k^{*}(z, w \bar{w} ; \lambda)$ is in $L^{2}(B)$. For, from (4.31) we have

$$
k^{*}(z, \bar{w} ; \lambda)=\sum_{n=0}^{\infty}\left[\frac{k_{n}^{1 / 2}\left(\phi_{n}(w)\right)^{-}}{1+\lambda k_{n}}\right] \phi_{n}(z) k_{n}^{-1 / 2}
$$

Now,

$$
\sum_{n=0}^{\infty}\left|\frac{k_{n}^{1 / 2}\left(\phi_{n}(w)\right)^{-}}{1+\lambda k_{n}}\right|^{2}<|\lambda|^{-2} \sum_{n=0}^{\infty} \frac{\left|\phi_{n}(w)\right|^{2}}{k_{n}}=|\lambda|^{-2} K_{B}(w, \bar{w})<\infty .
$$

From (4.31), (4.7), (4.9), and (4.10), we have a corresponding Fredholm development

$$
k^{*}(z, \bar{w} ; \lambda)=\sum_{n=0}^{\infty} \frac{A_{n}(z, \bar{w}) \lambda^{-n}}{n !} / \sum_{n=0}^{\infty} \frac{c_{n} \lambda^{-n+1}}{n !} .
$$

The modified kernel $k^{*}(z, \bar{w} ; \lambda)$ may be regarded as the resolvent kernel for the integral equation 


$$
\begin{aligned}
f(z) & =g(z)+\lambda^{-1} \iint_{G} K_{B}(z, \bar{w}) g(w) d A_{w} \\
& =\iint_{G} K^{*}(z, \bar{w} ; \lambda) g(w) d A_{w} ; \quad K^{*}=K_{G}+\lambda^{-1} K_{B}
\end{aligned}
$$

providing the solution is written in the form

$$
g(z)=f(z)-\iint_{G} k^{*}(z, \bar{w} ; \lambda) f(w) d A_{w} .
$$

The kernels $K$ and $k^{*}$ both contain the kernel functions $K_{G}$ and $K_{B}$. For we have immediately from (4.1)

$$
\begin{aligned}
K(z, \bar{w} ; 0) & =K_{G}(z, \bar{w}), \\
\lim _{\lambda \rightarrow \infty} \frac{1}{\lambda} K(z, \bar{w} ; \lambda) & =-K_{B}(z, \bar{w}),
\end{aligned}
$$

while from (4.31), (4.20), and (4.22)

$$
\begin{aligned}
k^{*}(z, \bar{w} ; 0) & =K_{G}(z, \bar{w}), \\
\lim _{\lambda \rightarrow \infty} \lambda k^{*}(z, \bar{w} ; \lambda) & =K_{B}(z, \bar{w}) .
\end{aligned}
$$

As regards the proof of (4.38), we have

$$
K_{B}(z, \bar{w})-\lambda k^{*}(z, \bar{w} ; \lambda)=\sum_{n=0}^{\infty} \frac{\phi_{n}(z)\left(\phi_{n}(w)\right)^{-}}{k_{n}\left(1+\lambda k_{n}\right)}
$$

so that by the Schwarz inequality

$$
\begin{aligned}
\left|K_{B}(z, \bar{w})-k^{*}(z, \bar{w} ; \lambda)\right|^{2} & \leqq \sum_{n=0}^{\infty} \frac{\left|\phi_{n}(z)\right|^{2}}{k_{n}^{2}} \sum_{n=0}^{\infty} \frac{\left|\phi_{n}(w)\right|^{2}}{\left(1+\lambda k_{n}\right)^{2}} \\
& \leqq K_{B}(z, \bar{z})|\lambda|^{-2} \sum_{n=0}^{\infty} \frac{\left|\phi_{n}(w)\right|^{2}}{\left(\lambda^{-1}+k_{n}\right)^{2}} \\
& \leqq|\lambda|^{-2} K_{B}(z, \bar{z}) K_{B}(w, \bar{w}) .
\end{aligned}
$$

With $z, w$ fixed in $B$, we now let $\lambda \rightarrow \infty$, and obtain (4.38).

5. An extremal problem for analytic functions. In the present section we consider the problem of approximation raised in the introduction. We shall assume that there have been given two regions $G$ and $B$, bounded, and with $\bar{G} \subset B$. We shall assume further that the set $\left\{\phi_{n}(z)\right\}$ of doubly orthogonal functions is complete for $L^{2}(G)$, or, less generally, that the regions $G$ and $B$ satisfy the sufficient geometric conditions given in $\$ 4$ which insure this completeness.

Let there be given a function $f \in L^{2}(G)$ and a positive number $M$. We wish to approximate $f$ by functions $f_{M}$ which are of class $L^{2}(B)$ and of $B$-norm 
not exceeding $M$ in such a way that $\left\|f-f_{M}\right\|_{G}=$ minimum. Introduce the set $\left\{\phi_{n}(z)\right\}$. Then we have

$$
f(z)=\sum_{n=0}^{\infty} a_{n} \phi_{n}(z) ; \quad a_{n}=\left(f, \phi_{n}\right)_{G}
$$

and similarly we may write for $f_{M}(z)$

$$
f_{M}(z)=\sum_{n=0}^{\infty} a_{n}^{(M)} \phi_{n}(z) ; \quad a_{n}^{(M)}=k_{n}^{-1 / 2}\left(f, \phi_{n} k_{n}^{-1 / 2}\right)_{B}
$$

Now

$$
\left\|f_{M}\right\|_{B}^{2}=\sum_{n=0}^{\infty}\left|a_{n}^{(M)}\right|^{2} k_{n}
$$

while we have

$$
\left\|f-f_{M}\right\|^{2}=\sum_{n=0}^{\infty}\left|a_{n}-a_{n}^{(M)}\right|^{2} .
$$

We now make the additional hypothesis (and without it our problem is solved trivially) that the given function $f(z)$ cannot be continued analytically so as to be regular in $B$ and of $B$-norm not exceeding $M$. This is to say, we are requiring that $\sum_{n=0}^{\infty}\left|a_{n}\right|{ }^{2} k_{n}>M^{2}$. Thus, in view of (5.3) and (5.4), our problem has been reduced to the minimal problem for the space $l^{2}$ already considered in \$2. We state our next theorem.

ThEOREM 4. Under the above assumptions as to the regions $G$ and $B$, the extremal problem for analytic functions considered here possesses one and only one solution. In terms of the doubly orthogonal functions $\phi_{n}(z)$, this solution is given explicitly by

$$
f_{M}(z)=f(z ; \lambda(M))=\sum_{n=0}^{\infty} \frac{a_{n} \phi_{n}(z)}{1+\lambda k_{n}}
$$

where $\lambda$ is the positive root of the equation

$$
\|f(z ; \lambda)\|_{B}^{2}=\sum_{j=0}^{\infty} \frac{\left|a_{j}\right|^{2} k_{j}}{\left(1+\lambda k_{j}\right)^{2}}=M^{2} .
$$

The measure of the approximation of $f_{M}$ to $f$ over $G$ is given by

$$
\Delta^{2}(f ; M)=\left\|f-f_{M}\right\|_{G .}^{2}=\lambda^{2} \sum_{j=0}^{\infty} \frac{\left|a_{j}\right|^{2} k_{j}^{2}}{\left(1+\lambda k_{j}\right)^{2}} .
$$

As $M \rightarrow \infty, \lambda \rightarrow 0$ through positive values, and we have 


$$
\lim _{\lambda \rightarrow 0} \Delta^{2}(f ; M(\lambda))=0,
$$

and this implies that

$$
\lim _{\lambda \rightarrow 0} f(z ; \lambda)=f(z)
$$

uniformly in any closed subregion $\bar{G}^{\prime} \subset G$.

Proof. We notice at first that $f(z ; \lambda)=\sum_{n=0}^{\infty} a_{n} k_{n}^{1 / 2} \phi_{n}^{*}(z) /\left(1+\lambda k_{n}\right)$. Now $\sum_{n=0}^{\infty}\left|a_{n} k_{n}^{1 / 2} /\left(1+\lambda k_{n}\right)\right|^{2} \ll|\lambda|-2 \sum_{n=0}^{\infty}\left|a_{n}\right|^{2}=|\lambda|-2|| f \|_{G}^{2}<\infty$, so that $f(z ; \lambda)$ $\in L^{2}(B)$. (5.5) follows from (2.15), (5.6) from (2.16), (5.7) from (2.18). (5.8) follows from (2.7), while (5.9) follows from (5.8) by a standard theorem. (Cf., for example, Walsh [10, p. 109].) A direct proof of this fact using the representation (5.5) may be given in the following way. We have

$$
\begin{aligned}
|f(z)-f(z ; \lambda)| & =\left|\sum_{n=0}^{\infty} \frac{\lambda a_{n} k_{n} \phi_{n}(z)}{1+\lambda k_{n}}\right| \\
& \leqq \lambda \sum_{n=0}^{N}\left|a_{n} k_{n} \phi_{n}(z)\right|+\sum_{N+1}^{\infty}\left|a_{n} \phi_{n}(z)\right| .
\end{aligned}
$$

Given a subregion $\bar{G}^{\prime} \subset G$ and $\epsilon>0$. In view of the absolute and uniform convergence of $\sum a_{n} \phi_{n}(z)$ in $\bar{G}^{\prime}$, we can, for $N$ sufficiently large, make $\sum_{N+1}^{\infty}\left|a_{n} \phi_{n}(z)\right|<\epsilon / 2$ for all $z \in \bar{G}^{\prime}$. Using this $N$, the sum $\sum_{0}^{N}\left|a_{n} k_{n} \phi_{n}(z)\right|$ has an upper bound $m$ for $z \in \bar{G}^{\prime}$. If we now set $\lambda_{0}=\epsilon / 2 m$, then for all $0<\lambda<\lambda_{0}$ we shall have $|f(z)-f(z ; \lambda)|<\epsilon, z \in \bar{G}^{\prime}$.

To each positive value of $\lambda$ and to each $f(z) \in L^{2}(G)$ there corresponds a family of functions of best approximation related to $f$ through (5.5) and whose $B$-norm may be computed by (5.6). It is therefore convenient to regard $\lambda$ as the independent parameter in the approximation process. In what follows we shall suppress $M$ in favor of $\lambda$, considering always $f(z ; \lambda)$ instead of $f_{M}(z)$.

The functions of best approximation $f(z ; \lambda)$ satisfy the following orthogonality relation:

COROLlaRY. Let $g(z)$ be any function of class $L^{2}(B)$ for which

$$
\iint_{B} f(z ; \lambda)(g(z))-d A=0 ;
$$

then we have

$$
\iint_{G}[f(z)-f(z ; \lambda)](g(z))-d A=0 .
$$

This follows from (5.5), (2.19), and (2.21).

The solution of the extremal problem given in the preceding section 
involves the set $\phi_{n}(z)$. By means of the resolvent kernel $k^{*}(z, \bar{w} ; \lambda)$ it is possible to obtain a representation of the extremal function which is in "directly computable" quantities and is independent of either the doubly orthogonal set or the corresponding eigenvalues $k_{n}$.

By way of a preliminary, we introduce the second iterate of the resolvent kernel $k^{*}$ by means of

$$
k^{*(2)}(z, \bar{w} ; \lambda)=\iint_{B} k^{*}(z, \bar{t} ; \lambda) k^{*}(t, \bar{w} ; \lambda) d A_{\imath} .
$$

It is to be noted that in (5.12) the composition has been carried out over $B$. From (4.31) it appears that $k^{*(2)}$ will have the expansion

$$
k^{*(2)}(z, \bar{w} ; \lambda)=\sum_{n=0}^{\infty} \frac{k_{n} \phi_{n}(z)\left(\phi_{n}(w)\right)^{-}}{\left(1+\lambda k_{n}\right)^{2}} .
$$

It is clear that for fixed $\lambda \neq 0, \neq-k_{n}^{-1}$ and for $w \in B$, the kernel $k^{*(2)}$ considered as a function of $z$ is of class $L^{2}(B)$. It may alternately be obtained from $k^{*}$ by means of a differentiation:

$$
k^{*(2)}(z, \bar{w} ; \lambda)=-\frac{\partial}{\partial \lambda} k^{*}(z, \bar{w} ; \lambda) .
$$

Theorem 5. Let $f(z) \in L^{2}(G)$. Then for each positive $\lambda$ the function

$$
f(z ; \lambda)=\iint_{G} k^{*}(z, \bar{w} ; \lambda) f(w) d A_{w}
$$

is of class $L^{2}(B)$ and is a function of best approximation to $f(z)$. Its B-norm is given by

$$
\begin{aligned}
\|f(z ; \lambda)\|_{B}^{2} & =\iint_{G} \iint_{G} k^{*(2)}(z, \bar{w} ; \lambda)(f(z))-f(w) d A_{z} d A_{w} \\
& =-\iint_{G} \iint_{G} \frac{\partial}{\partial \lambda} k^{*}(z, \bar{w} ; \lambda)(f(z))-f(w) d A_{z} d A_{w}
\end{aligned}
$$

while the measure of its approximation to $f$ over $G$ is given by

$$
\|f(z)-f(z ; \lambda)\|_{G .}^{2}=\iint_{G} \iint_{G} H(z, \bar{w} ; \lambda)(f(z))-f(w) d A_{z} d A_{w}
$$

where the kernel $H$ is defined by

$$
H(z, \bar{w} ; \lambda)=K_{G}(z, \bar{w})-k^{*}(z, \bar{w} ; \lambda)-\lambda k^{*(2)}(z, \bar{w} ; \lambda) .
$$

Proof. From (4.31) and (5.5) we arrive at (5.15) by termwise integration. Similarly, (5.16) is derived from (5.13) and (5.6). From (5.18), (4.22), (4.31), 
and (5.12) we have $\left.H(z, \bar{w} ; \lambda)=\sum_{n=0}^{\infty} \lambda^{2} k_{n}^{2} \phi_{n}(z) \phi_{n}(w)\right)^{-} /\left(1+\lambda k_{n}\right)^{2}$. Hence, in view of (5.7), we arrive at (5.17) by termwise integration.

COROLlary. The difference $g(z)=f(z)-f(z ; \lambda)$ satisfies the integral equation

$$
f(z)=g(z)+\lambda^{-1} \iint_{G} K_{B}(z, \bar{w}) g(w) d A_{w} .
$$

This follows from (5.15), (4.34), and (4.33).

Corollary. If the functions $A(z, w)$ and the constants $c_{n}$ are defined by (4.10), then we have

$$
f(z ; \lambda)=\sum_{n=0}^{\infty} \frac{\lambda^{-n}}{n !} \iint_{G} A_{n}(z, \bar{w}) f(w) d A_{w} \div\left(\sum_{n=0}^{\infty} \frac{c_{n} \lambda^{-n+1}}{n !}\right) .
$$

Proof. In view of (5.15) and (4.32) we have

$$
f(z ; \lambda)=\iint_{G}\left(\sum_{n=0}^{\infty} \frac{A_{n}(z, \bar{w})}{n !} \lambda^{-n}\right) f(w) d A_{w} \div\left(\sum_{n=0}^{\infty} \frac{c_{n} \lambda^{-n+1}}{n !}\right) .
$$

With $w$ confined to $G$ and fixed $z$ confined to $\bar{B}^{\prime} \subset B$ the inner series above will converge uniformly in $G$ (cf. the remarks following (4.10c)). We may therefore integrate termwise to arrive at (5.20).

The series (5.20) is valid for all $\lambda \neq 0, \neq-k_{n}^{-1}$, holding uniformly in $z$ for $z$ in $\bar{B}^{\prime} \subset B$, and provides a representation for the minimal functions directly in terms of integrals of known functions. Similar representations for the quantities $\|f(z ; \lambda)\|_{B}^{2}$ and $\|f(z)-f(z ; \lambda)\|_{G}^{2}$ may obviously be obtained from (4.32) through (5.16) and (5.17).

As $\lambda \rightarrow 0$ through positive values (corresponding to $M \rightarrow \infty$ ), we have $f(z ; \lambda) \rightarrow f(z)$ uniformly in every $\bar{G}^{\prime} \subset G$. Thus, referring to $(5.20)$ we have

$$
\lim _{\lambda \rightarrow+\infty} \sum_{n=0}^{\infty} \frac{\lambda^{n+1}}{n !} \iint_{G} A_{n}(z, \bar{w}) f(w) d A_{w} \div\left(\sum_{n=0}^{\infty} \frac{c_{n} \lambda^{n}}{n !}\right)=f(z) .
$$

As we have observed in $\S 4$, the functions $A_{n}(z, \bar{w})$ are certain linear combinations of $K_{B}(z, \bar{w}), \cdots, K_{B}^{(n)}(z, \bar{w})$, and hence the transformed functions

$$
A^{(n)} f(z)=\iint_{G} A_{n}(z, \bar{w}) f(w) d A_{w}
$$

are surely of class $L^{2}(B)$, and if the boundary of $B$ is analytic will be regular in a larger region.

This approach to an $f \in L^{2}(G)$ by means of its functions of best approximation and given by (5.21) possesses an interesting interpretation as a process of summation by integral means of the transformed functions $A^{(n)} f(z)$. The process of summation by integral means is a generalization of Borel's method 
of summation and may be defined as follows. (Cf., for example, Hardy [7, p. 79].)

Let there be given an entire function

$$
E(\lambda)=\sum_{n=0}^{\infty} p_{n} \lambda^{n}
$$

which is not a polynomial and has the further property that

$$
p_{n} \geqq 0
$$$$
(n=0,1, \cdots) \text {. }
$$

If we have

$$
\lim _{\lambda \rightarrow \infty} \sum_{n=0}^{\infty} p_{n} s_{n_{1}} \lambda^{n} \div E(\lambda)=s,
$$

then we shall say that the series $\sum_{n=0}^{\infty} s_{n}$ is summable $(E)$ to the value $s$ :

$$
\text { (E) } \sum_{n=0}^{\infty} s_{n}=s \text {. }
$$

Any $E$ method of summation is regular, that is, it sums to the appropriate value any convergent series. If $p_{n}=(n !)^{-1}$, the method becomes Borel's method of summation.

If we make the selection $p_{n}=c_{n} / n$ !, then from (4.9), $E(\lambda)=D(-\lambda)$. Since $\sum_{n=0}^{\infty} k_{n}^{-1}<\infty$, we know that $D(\lambda)$ has the product expansion (cf., for example, Goursat $[6$, p. 457])

$$
D(\lambda)=\prod_{n=0}^{\infty}\left(1-\frac{\lambda}{k_{n}}\right)
$$

so that $E(\lambda)=\prod_{n=0}^{\infty}\left(1+\lambda / k_{n}\right)$. This implies that

$$
p_{n}=\sum_{i_{1}<i_{2}<\cdots<i_{n}}\left(k_{i_{1}} k_{i_{2}} \cdots k_{i_{n}}\right)^{-1}>0 .
$$

We can also arrive at (5.28) through (4.12), noting that $a_{m}=\sum_{n=0}^{\infty} k_{n}^{-m}$. In the summation process described above, select $s_{n}=n A^{(n-1)} f(z) / c_{n}$ $(n=0,1, \cdots)$. Therefore from (5.25) and (5.21) we may write

$$
f(z)=(E) \sum_{n=0}^{\infty} \frac{n A^{(n-1)} f(z)}{c_{n}} .
$$

Each $f \in L^{2}(G)$ may thus be regarded as the $(E)$ sum of functions of class $L^{2}(B)$. The process $(E)$ is generated by the entire function $E(\lambda)=D(-\lambda)$. It depends merely upon the two regions $G, B$ and is independent of the function $f$ chosen. We have proved the following theorem.

THEOREM 6. Let $G$ and $B$ be two regions satisfying the conditions described 
previously. Then it is possible to set up a regular method of summation by integral means, that method depending only upon the regions $G, B$, such that every function of class $L^{2}(G)$ may be represented through (5.29) as the $(E)$ sum of functions of class $L^{2}(B)$. This process of summation is identical with the approach to $f \in L^{2}(G)$ of its functions of best approximation as $M \rightarrow \infty$.

Theorem 6 can be regarded as a kind of generalization of the Borel and Mittag-Leffler theorems on the summability of the Maclaurin expansion of regular functions.

If we make the particular selection $f(z)=K_{G}(z, \bar{w})$, w fixed in $G$, then by (5.22) we have $A^{(n)} f(z)=A(z, \bar{w})$, so that there is obtained

$$
K_{G}(z, \bar{w})=(E) \sum_{n=0}^{\infty} \frac{n A_{n-1}(z, \bar{w})}{c_{n}} .
$$

On the other hand, if the selection $f(z)=K_{B}(z, w)$ is made, then using (4.10b), (5.22), and (5.26),

$$
K_{B}(z, \bar{w})=(E) \sum_{n=0}^{\infty}\left\{K_{B}(z, \bar{w})-\frac{A_{n}(z, \bar{w})}{c_{n}}\right\} .
$$

6. Functions of best approximation. We shall say that a function $f \in L^{2}(B)$ is a function of best approximation (in the sense of our extremal problem) if there exists an $f^{*} \in L^{2}(G)$ and a constant $M$ such that under the condition $\|g\|_{B} \leqq M$, the expression $\left\|f^{*}-g\right\|_{G}$ is minimized by $g=f$. In the present section we investigate some properties of the class of functions of best approximation.

Let $f(z) \in L^{2}(G)$ and possess the Fourier expansion $f(z)=\sum_{n=0}^{\infty} a_{n} \phi_{n}(z)$; $\sum_{n=0}^{\infty}\left|a_{n}\right|^{2}<\infty$. We find easily from (3.1) and (4.20) that

$$
T f(z)=\sum_{n=0}^{\infty} \frac{a_{n} \phi_{n}(z)}{k_{n}} .
$$

More generally we have

$$
T^{(j)} f(z)=\sum_{n=0}^{\infty} \frac{a_{n} \phi_{n}(z)}{k_{n}^{j}} \quad(j=1,2, \cdots) .
$$

Using (6.2), we may extend the definition of the $j$ th iterate of $T$ to values of $j$ which are not positive integers. Reciprocally, given a function $h(z)$ $=\sum_{n=0}^{\infty} a_{n} \phi_{n}(z)$, it will be a $T^{(j)}$ transform of an $f \in L^{2}(G)$ if and only if

$$
\sum_{n=0}^{\infty}\left|a_{n}\right|^{2} k_{n}^{2 j}<\infty
$$

The class of $T^{(0)}$ transforms is $L^{2}(G)$. The class of $T^{(1 / 2)}$ transforms is 
$L^{2}(B)$. For assume that $h(z)=\sum_{n=0}^{\infty} a_{n} \phi_{n}(z)$ with $\sum_{n=0}^{\infty}\left|a_{n}\right|{ }^{2} k_{n}<\infty$. Then $h(z)=\sum_{n=0}^{\infty} a_{n} k_{n}^{1 / 2} \phi_{n}^{*}(z)$, so that in view of the Riesz-Fischer Theorem, $h(z)$ $\in L^{2}(B)$. The class of $T$ transforms coincides with the class of functions of best approximation, as we shall now show.

Theorem 7. A function $f(z) \in L^{2}(B)$ is a function of best approximation if and only if, for some $g \in L^{2}(G)$,

$$
f(z)=T g(z) .
$$

Proof. If $f(z)$ is of best approximation to $f^{*}(z)=\sum_{n=0}^{\infty} a_{n}^{*} \phi_{n}(z)$, then, by Theorem 4 , it must be of the form $f(z)=f^{*}(z ; \lambda)=\sum_{n=0}^{\infty} a_{n}^{*} \phi_{n}(z) /\left(1+\lambda k_{n}\right)$ for some positive $\lambda$. Now,

$$
\sum_{n=0}^{\infty} \frac{\left|a_{n}^{*}\right|^{2} k_{n}^{2}}{\left(1+\lambda k_{n}\right)^{2}} \leqq \lambda^{-2} \sum_{n=0}^{\infty}\left|a_{n}^{*}\right|^{2}<\infty .
$$

Hence by our remarks above, $f$ must be a $T$ transform. Conversely, a given $T g, g \in L^{2}(G)$, is of the form $\sum_{n=0}^{\infty} a_{n} \phi_{n}(z) / k_{n}, \sum_{n=0}^{\infty}\left|a_{n}\right|^{2}<\infty$. If we set $a_{n}^{\prime}=\left(a_{n} / k_{n}\right)\left(1+\lambda k_{n}\right)$, then $\left|a_{n}{ }^{\prime}\right| \leqq\left|a_{n}\right|(1+\lambda)$ so that $\sum_{n=0}^{\infty}\left|a_{n}^{\prime}\right|^{2}<\infty$. But $T g=\sum_{n=0}^{\infty} a_{n}^{\prime} \phi_{n}(z) /\left(1+\lambda k_{n}\right)$ and is therefore a best approximation to the function $\sum_{n=0}^{\infty} a_{n}^{\prime} \phi_{n}(z)$.

Corollary. The function $f(z)=\sum_{n=0}^{\infty} a_{n} \phi_{n}(z)$ is a function of best approximation if and only if

$$
\sum_{n=0}^{\infty}\left|a_{n}\right|^{2} k_{n}^{2}<\infty
$$

In a paragraph which follows we shall give a criterion which is independent of the doubly orthogonal functions.

The second half of the preceding theorem may be reworded as follows. Let a function $g \in L^{2}(G)$ be given. Then $T g$ is a best approximation to the oneparameter family of functions $L(g ; \lambda)$ given by

$$
L(g ; \lambda)=T g+\lambda g
$$$$
(\lambda \geqq 0) .
$$

Furthermore, the measure of the approximation over $G$ is given by

$$
\Delta=\lambda\|g\|_{G} .
$$

We next consider some particular functions of best approximations. Let $w$ be fixed in $B$. By (6.6) and (3.4), the kernel function $K_{B}(z, w \bar{w})$ is a best approximation to $\lambda K_{G}(z, \bar{w})+K_{B}(z, \bar{w})=\lambda K^{*}\left(z, \bar{w} ; \lambda^{-1}\right)$. This statement is equivalent to the identity

$$
\lambda \iint_{G} K^{*}\left(z, \bar{t} ; \lambda^{-1}\right) k^{*}(t, \bar{w} ; \lambda) d A_{\iota}=K_{B}(z, \bar{w}) .
$$


More generally, it is easy to show that $K_{B}^{(n)}(z, \bar{w})$ is the best approximation to $K_{B}^{(n)}(z, \bar{w})+\lambda K_{B}^{(n-1)}(z, \bar{w})(n=1,2, \cdots), \lambda \geqq 0$. Here $K_{B}^{(0)}$ is to be interpreted as $K_{G}$.

On the other hand, let $w$ be fixed in $G$. Then by the reproducing property of $K_{G}$ over $G$ we have

$$
\iint_{G} k^{*}(z, \bar{t} ; \lambda) K_{G}(t, \bar{w}) d A_{t}=k^{*}(z, \bar{w} ; \lambda)
$$

and, therefore, the resolvent kernel $k^{*}$ is, for $\lambda \geqq 0$, a best approximation to $K_{G}(z, \bar{w})$.

The Fredholm theory is rich in identities. Many of these can, by means of Theorem 5, be given interpretations as theorems on best approximation. Consider, for example, one of Fredholm's basic identities

$$
\begin{aligned}
\iint_{G} k^{*}\left(z, \bar{t} ; \lambda_{1}\right) k^{*}\left(t, \bar{w} ; \lambda_{2}\right) d A_{t} \\
=\left(\lambda_{1}-\lambda_{2}\right)^{-1}\left\{\lambda_{1} k^{*}\left(z, \bar{w} ; \lambda_{1}\right)-\lambda_{2} k^{*}\left(z, \bar{w} ; \lambda_{2}\right)\right\} \quad\left(\lambda_{1} \neq \lambda_{2}\right)
\end{aligned}
$$

and which in the Hermitian case at hand is readily verified through (4.31). This tells us that best approximations to the resolvent kernel $k^{*}$ are, for differing values of the parameter $\lambda$, appropriate combinations of $k^{*}$.

If we set $f(z)=\phi_{j}(z)(j=0,1, \cdots)$ in (5.5), there is obtained

$$
\phi_{j}(z ; \lambda)=\frac{\phi_{j}(z)}{1+\lambda k_{j}}=M \phi_{j}^{*}(z) .
$$

Thus, each doubly orthogonal function when multiplied by a suitable constant becomes its own best approximating function.

By combining Theorems 2 and 7, we arrive at the following result.

THEOREM 8. Let the region $B$ be bounded by a finite number of closed analytic curves. Then there exists a region $B^{*}$ with $\bar{B} C B^{*}$ such that each function of best approximation is regular in $B^{*}$. A region $B^{*}$ may be found which depends solely upon $G$ and $B$, and is independent of the particular function of class $L^{2}(G)$ which is being approximated.

We remark, therefore, that if $B$ has an analytic boundary, then regardless of the region $G$, no function of best approximation can possess a singularity on the boundary of $B$.

COROLlaRY. For an outer region $B$ with an analytic boundary, the doubly orthogonal functions are regular in $B^{*}$.

The following identity is easily derived from (6.1) and (5.5):

$$
T f(z)-f(z ; \lambda)=T f(z ; \lambda) .
$$


Let us designate the operation which produces $f(z ; \lambda)$ from $f(z)$ by $G_{\lambda}$, that is,

$$
G_{\lambda} f(z)=\iint_{G} k^{*}(z, \bar{w} ; \lambda) f(w) d A_{w} .
$$

Thus the operator $G_{\lambda}$ converts $f$ into a best approximation to $f$. We may now rewrite (6.11) in the following symbolic form

$$
T-\lambda G_{\lambda}=T G_{\lambda}
$$

whence

$$
G_{\lambda}=T /(T+\lambda I) ; \quad T=\lambda G_{\lambda} /\left(I-G_{\lambda}\right) .
$$

If the first fraction of (6.14) is expanded in ascending powers of $T$, there is obtained the Neumann series

$$
G_{\lambda}=\sum_{n=0}^{\infty} \frac{(-1)^{n} T^{n+1}}{\lambda^{n+1}}
$$

where $T^{(n)} f(z)$ is given by (6.2). The series (6.15) will be valid for values of $\lambda$ which are sufficiently large, but by introducing an appropriate method of summability such as Mittag-Leffler's, it becomes valid, under appropriate interpretation for all $\lambda>0$. We shall indicate briefly how this can be accomplished.

Consider the resolvent kernel $k(z, w \bar{w} ; \lambda)=D(z, \bar{w} ; \lambda) / D(\lambda)$. The entire function $D(\lambda)$ possesses zeros only at the points $\lambda=k_{i}(i=0,1, \cdots)$. Thus, for $z, w$ fixed in $B, k(z, \bar{w} ; \lambda)$ considered as a function of the complex variable $\lambda$ is regular in the star-region $R$ obtained by deleting the segment $S$ : (Im $\lambda$ $\left.=0 ; \operatorname{Re} \lambda \geqq k_{0}>1\right)$ from the complex $\lambda$-plane. Mittag-Leffler's method of summation will sum the series expansion of $k$, i.e., the series (4.6) for all $\lambda$ in $R$. We therefore have

$$
\lim _{\delta \rightarrow 0} \sum_{n=1}^{\infty} \frac{\lambda^{n-1} K_{B}^{(n)}(z, \bar{w})}{\Gamma(1+\delta(n-1))}=k(z, \bar{w} ; \lambda), \quad \lambda \in R .
$$

Comparing this with (4.31), we obtain

$$
k^{*}(z, \bar{w} ; \lambda)=\lim _{\delta \rightarrow 0} \sum_{n=1}^{\infty} \frac{(-1)^{n-1} K_{B}^{(n)}(z, \bar{w})}{\lambda^{n} \Gamma(1+\delta(n-1))} .
$$

It may be shown that for $z, w \in \bar{B}^{\prime} \subset B$, the limit in (6.16) and hence in (6.17) holds uniformly, so that from (5.5) and (6.17) there is obtained

(6.18) $f(z ; \lambda)=\lim _{\delta \rightarrow 0} \sum_{n=1}^{\infty} \frac{(-1)^{n-1}}{\Gamma(1+\delta(n-1)) \lambda^{n}} \iint_{G} K_{B}^{(n)}(z, \bar{w}) f(w) d A_{w}$ 
or symbolically,

$$
G_{\lambda}=(M) \sum_{n=1}^{\infty}(-1)^{n-1} T^{n} / \lambda^{n}
$$

where $(M)$ stands for the Mittag-Leffler sum of the series which follows. Furthermore, from (5.9) we obtain the representation

$$
f(z)=\lim _{\lambda \rightarrow \infty}(M) \sum_{n=1}^{\infty}(-1)^{n-1} \lambda^{n} T^{(n)} f(z) ; \quad f \in L^{2}(G) .
$$

In particular, if $f(z)=K_{G}(z, \bar{w})$, w fixed in $G$, we have $T^{(n)} f=K_{B}^{(n)}(z, \bar{w})$, so that

$$
K_{G}(z, \bar{w})=\lim _{\lambda \rightarrow \infty}(M) \sum_{n=1}^{\infty}(-1)^{n-1} \lambda^{n} K_{B}^{(n)}(z, \bar{w}) .
$$

We return now to (6.14). As special limiting values there may be obtained (cf. (4.35), (4.36), and (4.38))

$$
\begin{gathered}
\lim _{\lambda \rightarrow \infty} G_{\lambda}=0 ; \quad \lim _{\lambda \rightarrow 0} G_{\lambda}=I, \\
\lim _{\lambda \rightarrow \infty} \lambda G_{\lambda}=T .
\end{gathered}
$$

Inversion formulae for the operator $T$ may be found as follows. It is easily shown that for $f \in L^{2}(G)$ we have

$$
\iint_{B} k^{*}(z, \bar{w} ; \lambda) T f(w) d A_{w}=G_{\lambda} f(z)=f(z ; \lambda) .
$$

Introducing as operator $B_{\lambda}$ by

$$
\iint_{B} k^{*}(z, \bar{w} ; \lambda) g(w) d A_{w}=B_{\lambda} g ; \quad g \in L^{2}(B),
$$

then we have from (6.24)

$$
B_{\lambda} T=G_{\lambda}
$$

so that from (6.22) there is obtained

$$
\lim _{\lambda \rightarrow 0} B_{\lambda} T=I
$$

Thus finally

$$
T^{-1}=\lim _{\lambda \rightarrow 0} B_{\lambda}
$$

In terms of integrations over the inner region $G$, the following alternate inversion is also obtainable 


$$
T^{-1} f(z)=\lim _{\lambda \rightarrow 0} \iint_{G} k^{*(2)}(z, \bar{w} ; \lambda) f(w) d A_{w} .
$$

As a further application of these ideas, we now give a necessary and sufficient condition that a function which is originally defined over $G$ and of class $L^{2}(G)$ be continuable analytically to $B$ and be of class $L^{2}(B)$ there. (In this connection, see Bergman [3, p. 18] where a criterion involving infinite Hermitian forms related to the doubly orthogonal functions has been given.) We have already seen that the necessary and sufficient conditions for this is that $f$ be a $T^{(1 / 2)}$ transform, i.e., if $f(z)=\sum_{n=0}^{\infty} a_{n} \phi_{n}(z)$, then we must have $\sum_{n=0}^{\infty}\left|a_{n}\right|^{2} k_{n}<\infty$. In Theorem 9, we give a criterion which is related to the process of best approximation and is independent of the doubly orthogonal functions.

THEOREM 9. The function $f(z)$ of class $L^{2}(G)$ can be continued analytically to be of class $L^{2}(B)$ and with $B$-norm equal to $M<\infty$ if and only if

$$
\lim _{\lambda \rightarrow 0}\|f(z ; \lambda)\|_{B}^{2}=M^{2}
$$

or, what is equivalent, if and only if

$$
\lim _{\lambda \rightarrow 0} \iint_{G} \iint_{G} k^{*(2)}(z, \bar{w} ; \lambda)(f(z))^{-} f(w) d A_{z} d A_{w}=M^{2} .
$$

Proof. The equivalence of conditions (6.30) and (6.31) follows from (5.16). Using (5.13), we find that (6.31) is equivalent to the condition

$$
\lim _{\lambda \rightarrow 0} \sum_{n=0}^{\infty} \frac{\left|a_{n}\right|^{2} k_{n}}{\left(1+\lambda k_{n}\right)^{2}}=M^{2} \text {. }
$$

We shall now prove that (6.32) is equivalent to $\sum_{n=0}^{\infty}\left|a_{n}\right|^{2} k_{n}=M^{2}$, a condition which is obviously necessary and sufficient for $\|f\|_{B}=M$. We therefore assume, at first, that $\sum_{n=0}^{\infty}\left|a_{n}\right|^{2} k_{n}=M^{2}$. Consider

$$
\sum_{n=0}^{\infty}\left|a_{n}\right|^{2} k_{n}-\sum_{n=0}^{\infty} \frac{\left|a_{n}\right|^{2} k_{n}}{\left(1+\lambda k_{n}\right)^{2}}=\sum_{n=0}^{\infty} \frac{\left|a_{n}\right|^{2} k_{n}\left(2 \lambda k_{n}+\lambda^{2} k_{n}^{2}\right)}{\left(1+\lambda k_{n}\right)^{2}}=S(\lambda) .
$$

Let $\epsilon>0$ be given. Determine $N$ so large that $\sum_{N+1}^{\infty}\left|a_{n}\right|{ }^{2} k_{n}<\epsilon / 2$. Now $S(\lambda)$ $\leqq \sum_{n=0}^{N}\left(2 \lambda k_{n}+\lambda^{2} k_{n}\right)\left|a_{n}^{2}\right|^{2} k_{n} /\left(1+\lambda k_{n}\right)^{2}+\sum_{N+1}^{\infty}\left|a_{n}\right|^{2} k_{n}$. Now determine $\lambda_{0}$ so small that $1-1 /\left(1+\lambda k_{n}\right)^{2}<\epsilon /\left\{2 \sum_{n=0}^{N}\left|a_{n}\right|^{2} k_{n}\right\}$ for $n=0,1, \cdots, N$ and for $0 \leqq \lambda \leqq \lambda_{0}$. Then $S(\lambda) \leqq \epsilon$ for $0 \leqq \lambda \leqq \lambda_{0}$. Therefore (6.32) follows. Conversely, assume that (6.32) holds. For each $N$ we have

$$
\sum_{n=0}^{N} \frac{\left|a_{n}\right|{ }^{2} k_{n}}{\left(1+\lambda k_{n}\right)^{2}} \leqq \sum_{n=0}^{\infty} \frac{\left|a_{n}\right|{ }^{2} k_{n}}{\left(1+\lambda k_{n}\right)^{2}} .
$$


Therefore, allowing $\lambda \rightarrow 0$ in the above equation, we obtain $\sum_{n=0}^{N}\left|a_{n}\right|^{2} k_{n} \leqq M^{2}$, and allowing $N \rightarrow \infty$, we obtain $\sum_{n=0}^{\infty}\left|a_{n}\right|^{2} k_{n} \leqq M^{2}$. However, since $\sum_{n=0}^{\infty}\left|a_{n}\right|^{2} k_{n}>\sum_{n=0}^{\infty}\left|a_{n}\right|^{2} k_{n} /\left(1+\lambda k_{n}\right)^{2}$, by letting $\lambda \rightarrow 0$ there is obtained $\sum_{n=0}^{\infty}\left|a_{n}\right|{ }^{2} k_{n} \geqq M^{2}$. Thus, $\sum_{n=0}^{\infty}\left|a_{n}\right|{ }^{2} k_{n}=M^{2}$.

As remarked previously, a function $f$ is a function of best approximation if and only if it is a $T$ transform. By treating (5.13) and (6.5) similarly, we can obtain the following criterion which is independent of the doubly orthogonal functions.

THEOREM 10. $f(z)$ is a function of best approximation if and only if

$$
\lim _{\lambda \rightarrow 0} \iint_{B} \iint_{G} k^{*(2)}(z, \bar{w} ; \lambda)(f(z))^{-} f(w) d A_{z} d A_{w}<\infty .
$$

Theorems 9 and 10 possess interpretations in terms of a process of summation by integral means which are analogous to Theorem 6. From (5.14) and (4.32) we may obtain the following representation of $k^{*(2)}(z, \bar{w} ; \lambda)$ as the ratio of two entire functions of $\lambda$ :

$$
k^{*(2)}(z, \bar{w} ; \lambda)=\sum_{p=0}^{\infty} D_{p}(z, \bar{w}) \lambda^{-p} / \lambda^{2} \sum_{p=0}^{\infty} e_{p} \lambda^{-p}
$$

where

$$
D_{p}(z, \bar{w})=\sum_{m=0}^{p}(2 m-p+1) \frac{c_{p-m} A_{m}(z, \bar{w})}{m !(p-m) !}
$$

and where

$$
e_{p}=\sum_{m=0}^{p} \frac{c_{m} c_{p-m}}{m !(p-m) !}
$$

the constants $c_{p}$ having been previously defined in (4.10c). In view of the remarks following (4.10c) and the fact that $\sum_{n=0}^{\infty} c_{n} \lambda^{-n} / n$ ! is an entire function of $\lambda^{-1}$, it follows that for all $\lambda$, the numerator of (6.34) converges uniformly and absolutely for $z$, w confined to $\bar{B}^{\prime} \subset B$. We can therefore write

$$
\begin{aligned}
\iint_{G} \iint_{G} k^{*(2)}(z, \bar{w} ; \lambda)(f(z))-f(w) d A_{z} d A_{w} \\
=\sum_{n=0}^{\infty} \lambda^{-n} \iint_{G} \iint_{G} D_{n}(z, \bar{w})(f(z))-f(w) d A_{z} d A_{w} / \lambda^{2} \sum_{n=0}^{\infty} e_{n} \lambda^{-n}
\end{aligned}
$$

so that the criterion (6.31) becomes

$$
\lim _{\lambda \rightarrow \infty} \sum_{n=0}^{\infty} \lambda^{n+2} \iint_{G} \iint_{G} D_{n}(z, \bar{w})(f(z))^{-} f(w) d A_{z} d A_{w} / \sum_{n=0}^{\infty} e_{n} \lambda^{n}=M^{2},
$$


If, in the process of summability described by (5.25), we now set $p_{n}=e_{n}$ $(n=0,1, \cdots), p_{n} s_{n}=\iint_{G} \iint_{G} D_{n-2}(z, \bar{w})(f(z))-f(w) d A_{z} d A_{w}(n=2,3, \cdots)$, $s_{n}=0(n=0,1)$, then $(6.38)$ takes the form

$$
\text { (e) } \sum_{n=0}^{\infty} e_{n}^{-1} \iint_{G} \iint_{G} D_{n-2}(z, \bar{w})(f(z))-f(w) d A_{z} d A_{w}=M^{2} \text {. }
$$

The symbol $(e)$ refers to a process of summability by integral means using the entire function $e(\lambda)=\sum_{n=0}^{\infty} e_{n} \lambda^{n}$ as the averaging function. We have therefore established the following result: let $G$ and $B$ satisfy the usual conditions; then it is possible to specify a process of summation by integral means such that a function of class $L^{2}(G)$ is continuable analytically to $B$ and with $B$-norm $M$ if and only if (6.39) holds. The process $(e)$ as well as the sequence of kernels $D_{n}(z, v \bar{v})$ depend solely upon the two regions $G, B$. This process is identical to the convergence to $M$ of the $B$-norms of functions of best approximation to $f(z)$.

There are some special cases of Theorem 5 which provide interesting interpretations of the resolvent kernels $k^{*}$ and $k^{*(2)}$. Suppose initially that the region $G$ is simply-connected. Let $w$ be a fixed point lying in $G$. If $\phi(z, w)$ denoted by the function which maps $G$ conformally onto the unit circle in such a way that the point $w$ goes into the origin, then it is known (cf. Berg$\operatorname{man}[3])$ that

$$
\frac{\partial}{\partial z} \phi(z, w)=\frac{K_{G}(z, \bar{w})}{K_{G}(w, \bar{w})} .
$$

Referring now to (6.9) and (4.37), we obtain

$$
\iint_{G} k^{*}(z, \bar{t} ; \lambda) \frac{\partial}{\partial t} \phi(t, w) d A_{t}=\frac{k^{*}(z, \bar{w} ; \lambda)}{k^{*}(w, \bar{w} ; 0)} .
$$

We have therefore proved the following theorem.

THEOREM 11. For $w$ fixed in $G$, the normalized resolvent kernel $k^{*}(z, \bar{w} ; \lambda) / k^{*}(w, \bar{w} ; 0)$ is the function of best approximation to the derivative of the mapping function of $G$.

If $G$ is not simply-connected, then from (6.9), (5.15), and (3.5), we may say similarly that $k^{*}(z, \bar{w} ; \lambda)$ are the functions of best approximation to $(-2 / \pi)\left(\partial^{2} g_{G}(z, w) / \partial z \partial \bar{w}\right)$.

With $w$ fixed in $G$, let us make the selection $f(z)=\partial \phi(z, w) / \partial z$ in Theorem 9. The following theorem results.

TheOREM 12. The function $\partial \phi(z, w) / \partial z$ can be continued analytically so as to be of $B$-norm $M$ if and only if

$$
\lim _{\lambda \rightarrow 0} k^{*(2)}(w, \bar{w} ; \lambda)=M^{2}\left[K_{G}(w, \bar{w})\right]^{2} .
$$


Proof. We have

$\iint_{G} \iint_{G} k^{*(2)}(z, \bar{t} ; \lambda)\left(K_{G}(z, \bar{w})\right)-K_{G}(t, \bar{w}) d A_{z} d A_{t}=k^{*(2)}(w, \bar{w} ; \lambda)\left[K_{G}(w, \bar{w})\right]^{2}$

by the reproducing property of $K_{G}$ over $G$. The theorem now follows from (6.40) and (6.31).

We note further that $\partial \phi(z, w) / \partial z$ is of class $L^{2}(B)$ if and only if

$$
\lim _{\lambda \rightarrow 0} k^{*(2)}(w, \bar{w} ; \lambda)<\infty \text {. }
$$

This is equivalent to

$$
T^{-1} K_{G}(w, \bar{w})<\infty .
$$

If, in particular, the region $G$ has a nonanalytic boundary, then the limit (6.43) will be infinite for all $B$ such that $\bar{G} \subset B$. Extension of these remarks to the case of a multiply-connected $G$ may be made through (3.5).

7. Some special cases. In the present section, we give some examples where the doubly orthogonal functions are easily obtained. Let $G$ be the circle $|z|<r_{1}$, and $B$ the concentric circle $|z|<r_{2}, r_{2}>r_{1}$. The doubly orthogonal set for this pair of regions is given by

$$
\begin{aligned}
\phi_{n}(z) & =\left(\frac{n+1}{\pi}\right)^{1 / 2} z^{n} / r_{1}^{n+1} \quad(n=0,1, \cdots), \\
k_{n} & =\left(r_{2} / r_{1}\right)^{2 n+2} .
\end{aligned}
$$

We have immediately

$$
k^{*}(z, \bar{w} ; \lambda)=\frac{1}{\pi} \sum_{n=0}^{\infty} \frac{z^{n} \bar{w}^{n}}{r_{1}^{2 n+2}+\lambda r_{2}^{2 n+2}}
$$

and that if $f(z)$ has the representation

$$
f(z)=\sum_{n=0}^{\infty} a_{n} z^{n}
$$

then the functions of best approximation $f(z ; \lambda)$ are given by $\left({ }^{3}\right)$

$$
f(z ; \lambda)=\sum_{n=0}^{\infty} \frac{a_{n} r_{1}^{2 n+2} z^{n}}{r_{1}^{2 n+2}+\lambda r_{2}^{2 n+2}} .
$$

In this case there is obtained further

$$
\|f\|_{G}^{2}=\pi \sum_{n=0}^{\infty} \frac{\left|a_{n}\right|^{2} r_{1}^{2 n+2}}{n+1},
$$

(3) Cf. Walsh and Nilson [13, p. 250], recalling that in this paper line integrals were used. 


$$
\begin{aligned}
& \text { (7.5b) } \quad\|f(z ; \lambda)\|_{B}^{2}=M^{2}=\pi \sum_{n=0}^{\infty} \frac{\left|a_{n}\right|^{2} r_{2}^{2 n+2}}{(n+1)\left(1+\lambda\left(r_{2} / r_{1}\right)^{2 n+2}\right)^{2}}, \\
& \text { (7.5c) }\|f-f(z ; \lambda)\|_{a}^{2}=\pi \lambda^{2} \sum_{n=0}^{\infty} \frac{\left|a_{n}\right|^{2} r_{2}^{4 n+4}}{(n+1) r_{1}^{2 n+2}\left(1+\left(r_{2} / r_{1}\right)^{2 n+2}\right)^{2}} .
\end{aligned}
$$

From (6.1) we have

$$
T^{(j)} f(z)=\left(r_{1} / r_{2}\right)^{2 j} \sum_{n=0}^{\infty} a_{n}\left(r_{1}^{2 j} / r_{2}^{2 j}\right)^{n} z^{n}=\left(r_{1} / r_{2}\right)^{2 j} f\left(r_{1}^{2 j} z / r_{2}^{2 j}\right)
$$

and from (6.18) we obtain the representation

$$
f(z ; \lambda)=\lim _{\delta \rightarrow 0} \sum_{n=0}^{\infty} \frac{(-1)^{n-1}}{\Gamma(1+\delta(n-1)) \lambda^{n}}\left(r_{1} / r_{2}\right)^{2 n} f\left(r_{1}^{2 n} z / r_{2}^{2 n}\right) .
$$

It will be observed that if $f(z)$ is regular in $|z|<r$, then $f(z ; \lambda)$ will be regular in $|z|<r\left(r_{2} / r_{1}\right)^{2}$.

As a second example, and one which is not simply-connected, let $0<r_{1}$ $<r_{2}<r_{3}<r_{4}<\infty$. As $G$, take the annulus $r_{2}<|z|<r_{3}$, and as $B$, the annulus $r_{1}<|z|<r_{4}$. In this case, the doubly orthogonal functions are as follows.

$$
\begin{array}{rlrl}
\phi_{2 n}(z) & =\left[\frac{n+1}{\pi\left(r_{3}^{2 n+2}-r_{2}^{2 n+2}\right)}\right]^{1 / 2} z^{n} & (n=0,1, \cdots), \\
\phi_{2 n-1}(z) & =\left[\frac{n-1}{\pi\left(r_{2}^{-2 n+2}-r_{3}^{-2 n+2}\right)}\right]^{1 / 2} z^{-n} \quad(n=2,3, \cdots), \\
\phi_{1}(z) & =\left[\frac{1}{2 \pi \log \left(r_{3} / r_{2}\right)}\right]^{1 / 2} z^{-1} .
\end{array}
$$

The characteristic constants are given by

$$
\begin{aligned}
k_{2 n} & =\left(r_{4}^{2 n+2}-r_{1}^{2 n+2}\right) /\left(r_{3}^{2 n+2}-r_{2}^{2 n+2}\right) & & (n=0,1, \cdots), \\
k_{2 n-1} & =\left(r_{1}^{-2 n+2}-r_{4}^{-2 n+2}\right) /\left(r_{2}^{-2 n+2}-r_{3}^{-2 n+2}\right) & & (n=1,2, \cdots), \\
k_{1} & =\log \left(r_{4} / r_{1}\right) / \log \left(r_{3} / r_{2}\right) . & &
\end{aligned}
$$

If $f(z)$ possesses the Laurent expansion

$$
f(z)=\sum_{n=-\infty}^{+\infty} a_{n} z^{n}
$$

then the functions of best approximation have the form

$$
f(z ; \lambda)=\sum_{n=0}^{\infty} \frac{a_{n} z^{n}}{1+\lambda k_{2 n}}+\sum_{n=-1}^{-\infty} \frac{a_{n} z^{n}}{1+\lambda k_{2 n-1}} .
$$

Of this case, we shall merely observe that $k_{2 n} \sim\left(r_{4} / r_{3}\right)^{2 n+2}, k_{2 n-1} \sim\left(r_{2} / r_{1}\right)^{2 n-2}$, 
and this implies that if $f(z)$ is regular in the annulus $a<|z|<a^{\prime} ; a \leqq r_{2}, a^{\prime} \geqq r_{3}$, then all functions of best approximation will be regular in the annulus $a\left(r_{1} / r_{2}\right)^{2}<|z|<a^{\prime}\left(r_{4} / r_{3}\right)^{2}$.

These special cases for which explicit solutions have been found are extendable by means of conformal mapping. Let a region $G$ lying in the $z$-plane be mapped conformally onto a region $G^{*}$ lying in the $w$-plane by means of $w=\Im(z)$. Designate the inverse mapping function by $z=\theta(w)$. It is easily shown that $f(z) \in L^{2}(G)$ if and only if $f^{*}(w)=f(\theta(w)) \theta^{\prime}(w) \in L^{2}\left(G^{*}\right)$, and $\|f(z)\|_{G}=\left\|f^{*}(w)\right\|_{G^{*}}$. Furthermore, suppose that $\bar{G} \subset B$ is given, and the whole configuration is mapped conformally onto $G^{*}, B^{*}$. If $\phi_{n}(z)$ is the set of doubly orthogonal functions for $G, B$, then,

$$
\phi_{n}^{*}(w)=\phi_{n}(\theta(w)) \theta^{\prime}(w) \quad(n=0,1, \cdots)
$$

will be doubly orthogonal for $G^{*}, B^{*}$. Both sets of regions possess the same characteristic numbers:

$$
k_{n}=k_{n}^{*} \quad(n=0,1, \cdots) .
$$

It appears, then, that the whole theory of best approximation is conformally invariant, and indeed from (5.5), (7.12), and (7.13) we obtain

$$
f_{G, B}(z ; \lambda)=f_{G^{*}, B^{*}}^{*}(w ; \lambda), \quad \quad w=\Im(z) .
$$

The simplest configuration is obtained when $G^{*}$ and $B^{*}$ are images of two concentric circles $|z|<r_{1},|z|<r_{2}, 0<r_{1}<r_{2}<\infty$. In this case we have

$$
\begin{aligned}
\phi_{n}^{*}(w) & =((n+1) / \pi)^{1 / 2}(\theta(w))^{n} \theta^{\prime}(w) / r_{1}^{n+1}, \\
k_{n}^{*} & =\left(r_{2} / r_{1}\right)^{2 n+2} .
\end{aligned}
$$

In the general simply-connected case, we can, at least, map $B$ onto the unit circle so that the transformation $T$ is expressed very simply through (3.9).

8. Generalizations. In this final section, we shall discuss briefly some generalizations which can be made. In $\$ 4$, we introduced the basic assumption that the system $\phi_{n}(z)$ be complete over $L^{2}(G)$. Our reason for this is that we wished to approximate functions of $L^{2}(G)$ by those of class $L^{2}(B)$. Assuming completeness, the former class is comprised of those functions $f(z)$ $=\sum_{n=0}^{\infty} a_{n} \phi_{n}(z)$ with $\sum_{n=0}^{\infty}\left|a_{n}\right|^{2}<\infty$. In case of the incompleteness of $\left\{\phi_{n}(z)\right\}$ over $L^{2}(G)$, these latter functions form a subset $L_{I}^{2}(G)$. If we agree to operate only within $L_{I}^{2}(G)$, that is, if we deal with the problem of approximating functions of class $L_{I}^{2}(G)$ by functions of class $L^{2}(B)$, then our previous results will remain intact. If an incomplete kernel function of $G$ is defined by

$$
K_{G}^{I}(z, \bar{w})=\sum_{n=0}^{\infty} \phi_{n}(z)\left(\phi_{n}(w)\right)^{-},
$$


then the class $L_{I}^{2}(G)$ is comprised of those and only those functions which have a representation of the form

$$
f(z)=\iint_{G} K_{G}^{I}(z, \bar{w}) h(w) d A_{w} ; \quad h(w) \in L^{2}(G) .
$$

This class may be characterized independently of the system $\left\{\phi_{n}(z)\right\}$ as follows: $f \in L_{I}^{2}(G)$ if and only if

$$
f(z)=\lim _{\lambda \rightarrow 0} \iint_{G} k^{*}(z, \bar{w} ; \lambda) h(w) d A_{w}
$$

for some $h(w) \in L^{2}(G)$.

The following modification of the original problem may also be contemplated. Instead of discussing complex-valued functions which are analytic in $G$, we may discuss real-valued functions which are harmonic in $G$, or, more generally, functions $u(x, y)$ which satisfy a partial differential equation of elliptic type

$$
\frac{\partial^{2} u}{\partial x^{2}}+\frac{\partial^{2} u}{\partial y^{2}}-q(x, y) u=0, \quad q(x, y) \geqq 0 \text { in } \bar{G} .
$$

If a scalar product is defined by

$$
E(u, v)=\iint_{G}\left[\frac{\partial u}{\partial x} \frac{\partial v}{\partial x}+\frac{\partial u}{\partial y} \frac{\partial v}{\partial y}+q u v\right] d x d y,
$$

the space of solutions $u$ for which $E(u, u)<\infty$ is a Hilbert space possessing a reproducing kernel $K_{G}(x, y ; \xi, \eta)$ (see Bergman and Schiffer [4]). For two regions $\bar{G} \subset B$, the integral equation

$$
\phi(x, y)=\lambda E\left[K_{B}(x, y ; \xi, \eta), \phi(\xi, \eta)\right]
$$

possesses eigenvalues $\phi_{n}(x, y)$ which are doubly orthogonal over $G$ and $B$ with respect to the metric (8.5). Much of the present theory will carry over to this more general situation.

\section{BIBLIOGRAPHY}

1. S. Bergman, Über die Entwicklung der harmonischen Funktionen der Ebene und des Raumes nach Orthogonalfunktionen, Math. Ann. vol. 96 (1922) pp. 237-271.

2. - Zwei Sätze ïber Funktionen von zwei komplexen Veränderlichen, Math. Ann. vol. 100 (1928) pp. 399-410.

3. - The kernel function and conformal mapping, Mathematical Surveys, no. 5, New York, American Mathematical Society, 1950.

4. S. Bergman and M. Schiffer, Kernel functions in the theory of partial differential equations of elliptic type, Duke Math. J. vol. 15 (1948) pp. 535-566.

5. O. J. Farrell, On approximation to an analytic function by polynomials, Bull. Amer. Math. Soc. vol. 40 (1934) pp. 908-914. 
6. E. Goursat, Cours d'analyse, vol. 3, Paris, 1923.

7. G. H. Hardy, Divergent series, Oxford, 1949.

8. E. N. Nilson and J. L. Walsh, Interpolation and approximation by functions analytic and bounded in a given region, Trans. Amer. Math. Soc. vol. 55 (1944) pp. 53-67.

9. M. Schiffer, The kernel function of an orthonormal system, Duke Math. J. vol. 13 (1946) pp. 529-540.

10. J. L. Walsh, Interpolation and approximation by rational functions in the complex domain, Amer. Math. Soc. Colloquium Publications, vol. 20, New York, 1935.

11. - On interpolation and approximation by functions analytic and bounded in a given region, Proc. Nat. Acad. Sci. U.S.A. vol. 24 (1938) pp. 477-486.

12. - - Taylor series and approximation to functions, Bull. Amer. Math. Soc. vol. 52 (1946) pp. 572-579.

13. J. L. Walsh and E. N. Nilson, On functions analytic in a region: approximation in the sense of least pth powers, Trans. Amer. Math. Soc. vol. 65 (1949) pp. 239-258.

HARVARD UNIVERSITY,

Cambridge, Mass. 